\title{
CRUISE RESULTS
}

\section{FISHERIES ECOSYSTEMS ASSESSMENT MARMAP SURVEY}

\section{Northeast Continental Shelf Ecosystem}

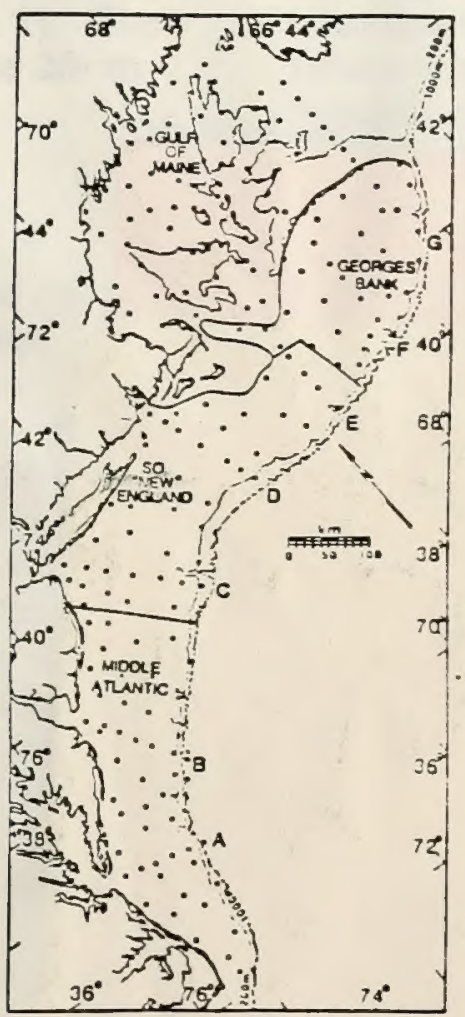

19 July to 2 August 1991

National Oceanographic and Atmospheric Administration National Marine Fisheries Service

Northeast Fisheries Center

Woods Hole, Massachusetts

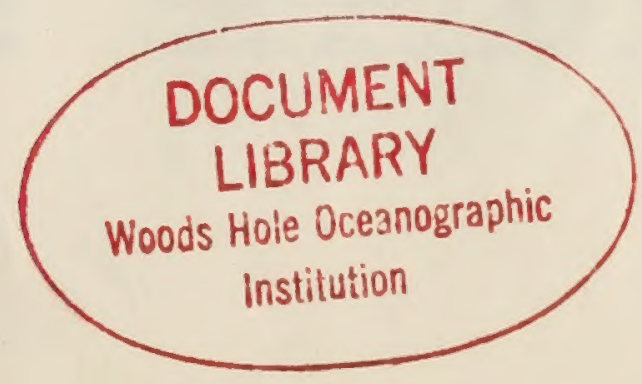



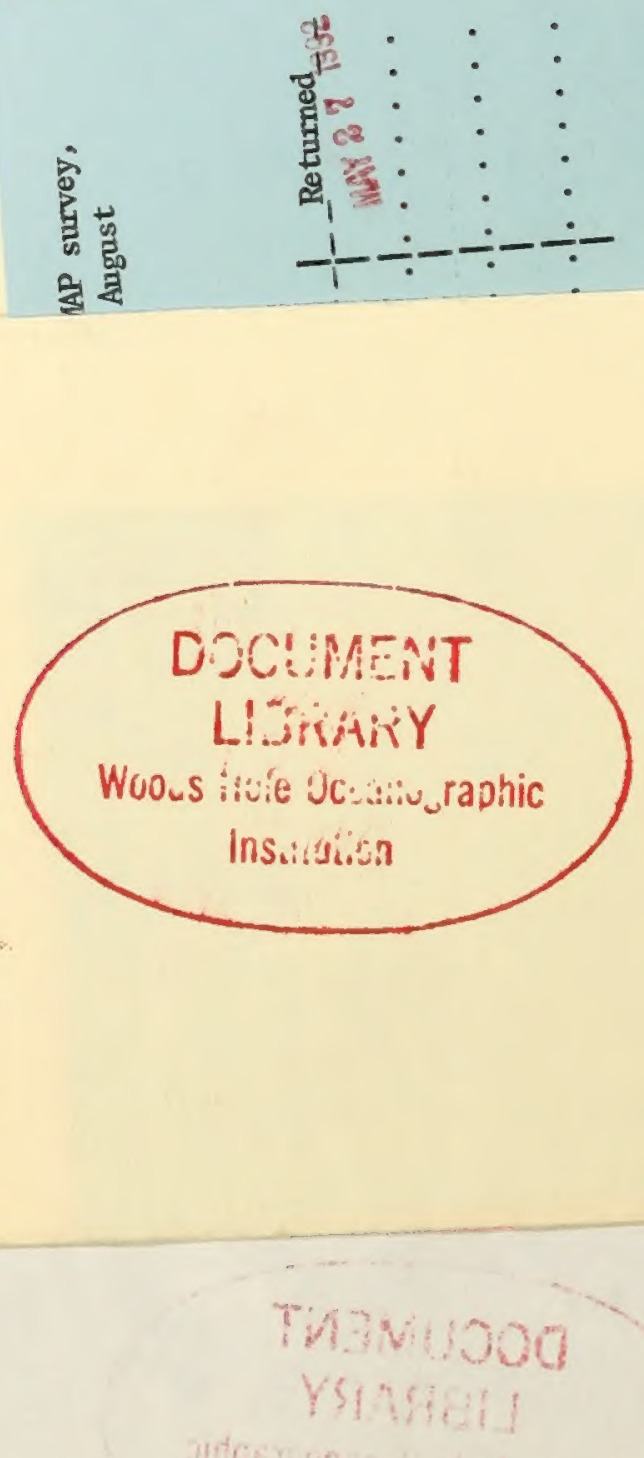
CRUISE RESULTS

R/V ARGO MAINE

Cruise AM 91-01

Fisheries Ecosystems Assessment--MARMAP Survey

\section{CRUISE PERIOD AND AREA}

The cruise period was from 19 July to 2 August, 1991. The area of operations was the Northeast Shelf Ecosystem from Cape Hatteras, North Carolina to Nova Scotia (Figure 1). The primary depth zone was from the 20- to 2,000-meter isobaths.

\section{OBJECTIVES}

This cruise was the first of the resumed MARMAP cruises termed Fisheries Ecosystem Assessment-MARMAP Surveys, the objective of which is a assess the impact of changing biological and physical properties of the NE Continental Shelf ecosystem which influence the sustainable productivity of the living marine resources. The JulyAugust survey was designed to measure spatial and temporal changes in the productivity of the waters of the NE Shelf ecosystem, and to index the changes in the ichthyoplankton and zooplankton communities. Key parameters measured were: water column temperatures, salinities, chlorophyll and nutrient concentrations; light transmission, ichthyo- and zooplankton composition, abundance and distribution; underway near surface chlorophyll concentration; and standard weather observations. This cruise initiated a reduced level of station coverage compared to earlier MARMAP surveys (1977-1986) from an average of 105 to 70 stations. An evaluation of the effectiveness of this strategy was one of the cruise's objectives. The major alteration to the 1977-1987 sampling protocol included towed-body sampling with the Continuous Plankton Recorder (CPR) between station transects; as well as along new transects. The key station transects were located off the major estuaries and across areas of particular importance (e.g., Georges Bank and the Gulf of Maine) and surface chlorophyll measurements along the entire track of the cruise.

Staff at the Northeast Fisheries Center Narragansett Laboratory will work jointly with scientists at several institutions in the analyses of the data as follows: (a) chlorophyil, nutrient, and light transmission--Bigelow Laboratory for Ocean Sciences, Boothbay Harbor, Maine; (b) water column temperature and salinity--Northeast Fisheries Center, Woods Hole, Massachusetts;

(c) phytoplankton and zooplankton-Sorting Centers, Szczecin and Gdynia, Poland; (d) ichthyoplankton--Northeast Fisheries Center, Sandy Hook, New Jersey; (e) meteorological data--National Oceanic and Atmospheric Administration, National Weather Service, Washington, D.C.

\section{METHODS}

Plankton sampling gear consisted of a 0.61-meter bongo frame fitted with 0.505 -millimeter and 0.333 -millimeter mesh nets. A 45-kilogram ball was attached beneath the frame to depress the sampler, and a bathykymograph was 
attached above the frame to record sampling depth throughout the tow. Digital flow meters were suspended within the mouths of the bongo samplers. Towing wire metering was employed, but no wire angle inclinometers were used. Tow speeds of approximately 2 knots were maintained by engaging and disengaging the vessel's main drive. Plankton tows were made at all stations along transects $A$ through $G$ (Figure 1).

The Hardy Continuous Plankton Recorder (CPR) was towed at a standard depth of 10 meters. Towing speed was 9 knots, and mesh aperture was $225 \times 234$ microns, when wet. A continuous record of the zooplankton and larger phytoplankton was thus obtained along transects $\mathrm{L}$ through $\mathrm{Q}$ (Figure 2).

A CTD with rosette, fluorometer, and transmissometer was deployed to near bottom or a maximum depth of 250 $\mathrm{m}$ at all stations along transect $\mathrm{A}$ through G. Temperature, salinity, fluorescence, and light transmission data were pressure averaged by one meter. Water samples were taken at up to six standard levels $(2 \mathrm{~m}, 10 \mathrm{~m}, 30 \mathrm{~m}, 50 \mathrm{~m}$, $75 \mathrm{~m}$, and near bottom) depth permitting, and samples were frozen for subsequent nutrient analysis. Samples to the standard depth of $75 \mathrm{~m}$ were filtered and frozen for chlorophyll calibration purposes, from one inshore, one midshelf, and one offshore station on each transect.

Continuous measurement of fluorescence at a depth of $2 \mathrm{~m}$ was made using a flow-through fluorometer connected in line with the ship's sea water system for the entire cruise track. During CPR towing no less than two, water samples were taken from a depth of $2 \mathrm{~m}$, filtered, and frozen per day for calibration purposes. Two-meter depth water samples were taken at the ends of the station transect for calibration purposes. Surface temperature was determined at each station with a stem thermometer and bucket.

At each station the bottom depth, wind speed and direction, air temperature, wet bulb temperature, atmospheric pressure, cloud type and amount, and wave height were recorded.

\section{RESULTS}

A summary of the measurements accomplished is given in Table 1. Continuous plankton records were obtained from 944 of the 1,000 desired miles. This difference was due to the entangling of a sandbar shark in the drive mechanism of the CPR. All 70 of the desired bongo tows were made. CTD, nutrient and fluorescence data were obtained from 66 of the 70 planned stations. The power supply to the CTD and rosette failed with 10 stations to go, forcing a switch to a self contained CDT and individual water bottles. Underway, 2 -m fluorescence data were obtained from the entire cruise track.

Preliminary processing of digital data was done on board and plots of temperature, salinity, sigma-t, fluorescence, and light transmission were prepared. Preliminary plots are given in Appendix I. They are presently undergoing quality control, and are, in some cases, displayed in relative units. Thus, they should be interpreted accordingly.

Several conclusions can be drawn from the preliminary examination of the cruise results:

(a) A short station transect should be added from the vicinity of Provincetown, Massachusetts, to Portland, Maine, to fill a gap in the station array; 
(b) XBT and surface salinity deployments between the station transects (along the towing transects) would greatly add to the utility of the survey temperature and salinity coverage;

(c) Given the instrumentation available on this cruise, such a survey could be accomplished with two persons per watch;

(d) The Northeast Fisheries Center's equipment supply, of the kind used on this cruise, is in need of repair and some replenishment, particularly with regard to rosette, water bottles, and flow-throw fluorometer;

(e) Fourteen to 17 days seems reasonable for the completion of such a cruise of the ALBATROSS IV, and usual weather.

\section{SPECIAL NOTES}

On 21 July the ARGO MAINE offered assistance to a 24-foot recreational fishing boat which was disabled some 60 miles off the North Carolina coast. Attempts to repair their engine by the Chief Engineer failed. The ARGO MAINE stood by for approximately 7 hours until a Coast Guard cutter out of Cape Hatteras arrive to tow in the disabled boat.

A port call of approximately $30-$ minutes at Narragansett, Rhode Island, the night of 26 July to pick up the license to conduct the cruise in Canadian waters.

\section{DISPOSITION OF DATA}

Biological samples from the CPR were delivered to the Narragansett, Rhode Island, Laboratory for preprocessing and forwarding to Gdynia, Poland, for identification of phytoplankton and zooplankton components. Samples from the $0.61-\mathrm{m}$ bongo were delivered to the Narragansett Laboratory for shipment to Zaklad Sortowania i Oznaczania Planktonu (ZSIOP), Szczecin, Poland, for volume determinations, sorting, identification and enumeration of plankton community structure and abundance. Chlorophyll and nutrient samples were delivered to the Narragansett Laboratory for preprocessing and forwarding to the University of Rhode Island for final analysis. Water column fluorescence and light transmission, and 2-m fluorescence data were delivered to the Narragansett Laboratory for processing. The final fluorescence and nutrient data will be forwarded to the Bigelow Laboratory for analyses. CTD data were delivered to the Narragansett Laboratory for preprocessing and forwarding to the Northeast Fisheries Center, Oceanography Branch, Woods Hole, for analysis.

\section{SCIENTIFIC PERSONNEL}

National Marine Fisheries Service, NEFC, Narragansett, RI

Jack Jossi, Chief Scientist

Julien Goulet

Thomas McKenney

Thomas Pothoff

Jerry Prezioso

Maine Maritime Academy, Castine ME

George Gatcomb

For further information contact: Jack W. Jossi, National Marine Fisheries Service, Northeast Fisheries Center, Narragansett, RI 02882.

Telephone-(401)782-3274. 
Table 1. Summary of Station Activities.

\begin{tabular}{|c|c|c|c|c|c|c|c|c|c|c|c|c|c|}
\hline \multirow[t]{3}{*}{ sta } & \multirow[t]{3}{*}{$\begin{array}{l}\text { Tran- } \\
\text { sect }\end{array}$} & \multicolumn{12}{|c|}{$\begin{array}{l}\text { Station Activities } \\
\text { umber of measurements }\end{array}$} \\
\hline & & \multicolumn{7}{|c|}{ Water Column } & \multirow{2}{*}{\multicolumn{2}{|c|}{$\begin{array}{l}\text { Surface } \\
\text { CHL SAL }\end{array}$}} & \multicolumn{3}{|c|}{ Ten-Meter } \\
\hline & & $6 \mathrm{~B} 3$ & $6 \mathrm{~B} 5$ & CTD & NUT & CHL & FLU & TRN & & & $\begin{array}{l}\text { CPR } \\
\text { SHT }\end{array}$ & $\begin{array}{l}\text { CPR } \\
\text { ALT }\end{array}$ & $\begin{array}{l}\text { CPR } \\
\text { HAU }\end{array}$ \\
\hline 1 & L & & & & & & & & & & c & & \\
\hline 2 & L & & & & & & & & c & & & c & \\
\hline 3 & L & & & & & & & & c & & & & \\
\hline 4 & L & & & & & & & & c & & & c & \\
\hline 5 & L & & & & & & & & c & & & & 339 \\
\hline 6 & A & c & c & c & 6 & 5 & c & c & c & & & & \\
\hline 7 & A & c & c & c & 6 & & c & c & c & & & & \\
\hline 8 & A & c & c & c & 4 & 3 & c & c & c & & & & \\
\hline 9 & A & c & C & C & 3 & & c & c & c & & & & \\
\hline 10 & A & c & c & C & 3 & & c & c & c & & & & \\
\hline 11 & A & c & c & c & 3 & & c & c & c & & & & \\
\hline 12 & A, M & c & c & c & 2 & 2 & C & c & c & & c & & \\
\hline 13 & $B, M$ & c & c & C & 6 & 5 & c & c & c & 1 & & & 95 \\
\hline 14 & B & c & c & c & 6 & & c & c & c & & & & \\
\hline 15 & B & c & c & c & 6 & & c & c & c & & & & \\
\hline 16 & B & c & c & c & 4 & 3 & c & c & c & & & & \\
\hline 17 & B & c & c & c & 4 & & c & c & c & & & & \\
\hline 18 & B & c & c & c & 3 & & c & c & c & & & & \\
\hline 19 & B & c & c & c & 3 & & c & c & c & & & & \\
\hline 20 & $B, N$ & c & c & c & 2 & 2 & c & c & c & 1 & $c$ & & \\
\hline 21 & $\mathrm{~N}$ & & & & & & & & c & & & c & \\
\hline 22 & $C, N$ & c & c & c & 6 & 5 & c & c & c & 1 & & & 129 \\
\hline 23 & C & C & c & c & 6 & & C & c & C & & & & \\
\hline 24 & C & C & c & c & 6 & & C & C & C & & & & \\
\hline 25 & C & c & c & c & 5 & & c & c & c & & & & \\
\hline 26 & C & c & C & C & 5 & 5 & C & C & C & & & & \\
\hline 27 & C & c & c & c & 4 & & C & c & c & & & & \\
\hline 28 & C & C & C & C & 3 & & C & c & c & & & & \\
\hline 29 & $c, 0$ & C & C & C & 3 & 2 & c & c & C & 1 & c & & \\
\hline 30 & 0 & & & & & & & & C & & & C & \\
\hline 31 & $D, O$ & c & c & C & 6 & 6 & c & c & c & 1 & & & 150 \\
\hline 32 & D & c & c & c & 6 & & c & C & C & & & & \\
\hline 33 & D & c & c & c & 6 & & c & c & c & & & & \\
\hline 34 & D & c & c & c & 6 & & c & c & c & & & & \\
\hline 35 & D & c & c & c & 5 & 3 & c & c & c & & & & \\
\hline 36 & D & c & c & c & 4 & & c & c & C & & & & \\
\hline 37 & D & c & c & c & 4 & & c & C & C & & & & \\
\hline 38 & D & c & C & C & 3 & 2 & c & c & c & 1 & & & \\
\hline 39 & P & & & & & & & & c & & c & & \\
\hline 40 & $\mathbf{P}$ & & & & & & & & C & & & c & \\
\hline 41 & P & & & & & & & & c & & & c & \\
\hline 42 & P & & & & & & & & c & & & C & \\
\hline 43 & P & & & & & & & & C & & & C & \\
\hline 44 & P & & & & & & & & c & & & C & \\
\hline 45 & $\mathbf{P}$ & & & & & & & & C & & & c & \\
\hline
\end{tabular}




\begin{tabular}{|c|c|c|c|c|c|c|c|c|c|c|c|c|c|}
\hline sta & $\mid \begin{array}{l}\text { Tran- } \\
\text { sect }\end{array}$ & wit & & ambe & atio & $\begin{array}{l}\text { on } A \\
\text { mea: }\end{array}$ & tivi & $\begin{array}{l}\text { ities } \\
\text { ients }\end{array}$ & or $s$ & sample & es per & sta & $\operatorname{tion}^{1}$ \\
\hline & & & ater & {$[\mathrm{COl}$} & Lumn & & & & Sur & eface! & Ter & 1-Met & er \\
\hline & & $6 \mathrm{B3}$ & $6 B 5$ & CTD & NUT & CHL & FLU & TRN & $\mathrm{CHL}$ & SAL & $\begin{array}{l}\text { CPR } \\
\text { SHT }\end{array}$ & $\begin{array}{l}\text { CPR } \\
\text { ALT }\end{array}$ & $\begin{array}{l}\text { CPR } \\
\text { HAU }\end{array}$ \\
\hline 46 & $P, Q$ & & & & & & & & c & & C & & 225 \\
\hline 47 & $Q$ & & & & & & & & c & & & C & 135 \\
\hline $\begin{array}{l}48 \\
49\end{array}$ & $\stackrel{Q}{G}$ & $c$ & c & c & 5 & 5 & c & C & $\begin{array}{l}c \\
c\end{array}$ & & & & 135 \\
\hline 50 & G & c & c & c & 5 & & c & c & c & & & & \\
\hline 51 & G & C & C & C & 6 & ( & c & $c$ & c & & & & 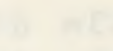 \\
\hline 52 & G & c & c & c & 5 & $a^{2}$ & c & c & c & & & & 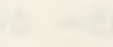 \\
\hline 53 & G & C & c & c & 5 & & C & C & C & & & 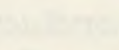 & 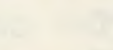 \\
\hline 54 & G & C & C & C & 6 & 5 & C & C & c & 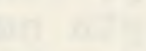 & & & 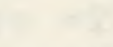 \\
\hline 55 & G & C & C & c & 6 & & C & C & c & & & & 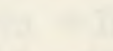 \\
\hline 56 & G & C & c & c & 6 & & C & c & c & & & & 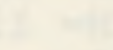 \\
\hline 57 & G & c & $\mathrm{c}$ & c & 5 & 5 & c & c & c & 1 & & & 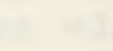 \\
\hline 58 & G & c & c & c & 6 & & c & c & c & & & & \\
\hline 59 & G & c & $c$ & c & 6 & & c & c & $c$ & & & & . \\
\hline 60 & G & c & c & c & 6 & & c & c & C & 1 & & & 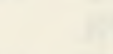 \\
\hline 61 & F & C & c & c & 6 & & c & $c$ & $c$ & 1 & & & 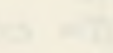 \\
\hline 62 & F & c & c & c & 6 & & C & c & c & & & & \\
\hline 63 & F & c & c & c & 5 & 5 & C & C & C & & & & \\
\hline 64 & F & c & c & c & 5 & & C & C & C & & & & \\
\hline 65 & F & c & $c$ & c & 3 & & c & C & c & a & & t & 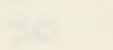 \\
\hline 66 & F & C & C & C & 4 & 3 & c & c & C & & & & \\
\hline 67 & F & C & c & C & 3 & & c & c & C & & & & \\
\hline 68 & F & c & C & c & 6 & 5 & C & C & c & & & & \\
\hline 69 & F & C & c & c & 6 & & c & c & c & & & & \\
\hline 70 & $F$ & C & C & c & 6 & & c & c & c & & & & \\
\hline 71 & F & c & C & C & 6 & & C & C & c & & & & \\
\hline 72 & F & c & c & C & 6 & & $\mathrm{c}$ & $c$ & c & & & & \\
\hline 73 & F & C & c & c & 5 & 4 & c & $c$ & c & 1 & & & \\
\hline 74 & $\mathrm{E}$ & c & c & c & 6 & 5 & c & c & C & 1 & & & \\
\hline 75 & $\mathrm{E}$ & c & c & c & 6 & & C & C & c & & & & \\
\hline 76 & $\mathrm{E}$ & c & C & & & & & & c & 1 & & & \\
\hline 77 & E & C & c & c & 4 & 4 & & & C & 2 & & & \\
\hline 78 & $\mathrm{E}$ & c & c & & & & & & c & 1 & & & \\
\hline 79 & $\mathrm{E}$ & c & c & c & 4 & 4 & & & C & $\perp$ & & & \\
\hline 80 & $E$ & c & C & & & & & & c & 1 & & & \\
\hline 81 & $\mathrm{E}$ & c & C & c & 4 & 4 & & & C & 1 & & & \\
\hline 82 & $\mathrm{E}$ & C & c & 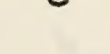 & & & & & C & $\begin{array}{l}1 \\
1\end{array}$ & & & \\
\hline 83 & $\mathbf{E}$ & c & c & c & 4 & 4 & & & $c$ & $\frac{1}{1}$ & & & \\
\hline 84 & $\bar{E}$ & C & c & c & $\begin{array}{l}4 \\
4\end{array}$ & 4 & & & c & 1 & & & \\
\hline 85 & $\mathrm{E}$ & C & c & $\begin{array}{l}\text { C } \\
\text { c }\end{array}$ & 4 & & & & c & 1 & & & \\
\hline 86 & $\mathrm{E}$ & c & c & c & 4 & 4 & & & C & 1 & & & \\
\hline 87 & E & c & $\begin{array}{l}c \\
c\end{array}$ & C & 4 & & & & C & 1 & & 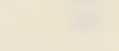 & \\
\hline & $\mathbf{E}$ & c & C & c & 4 & & & & C & 4 & & & \\
\hline
\end{tabular}




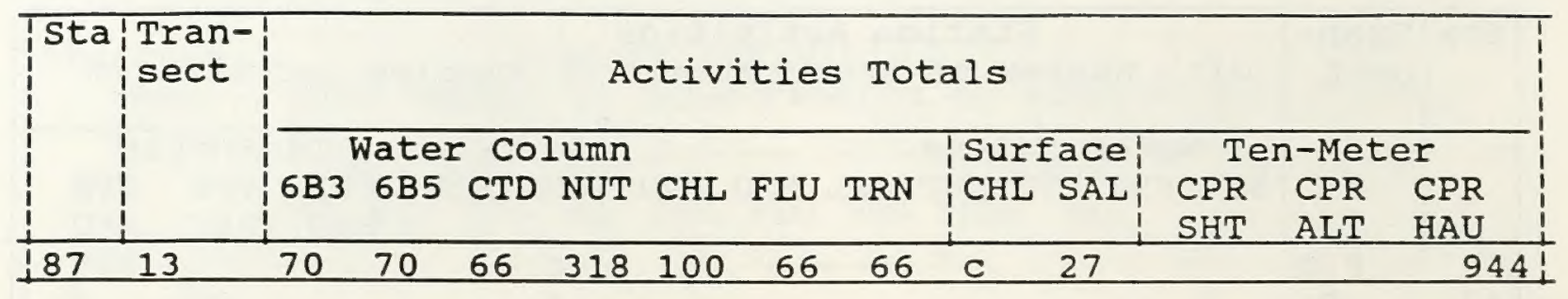

Legend:

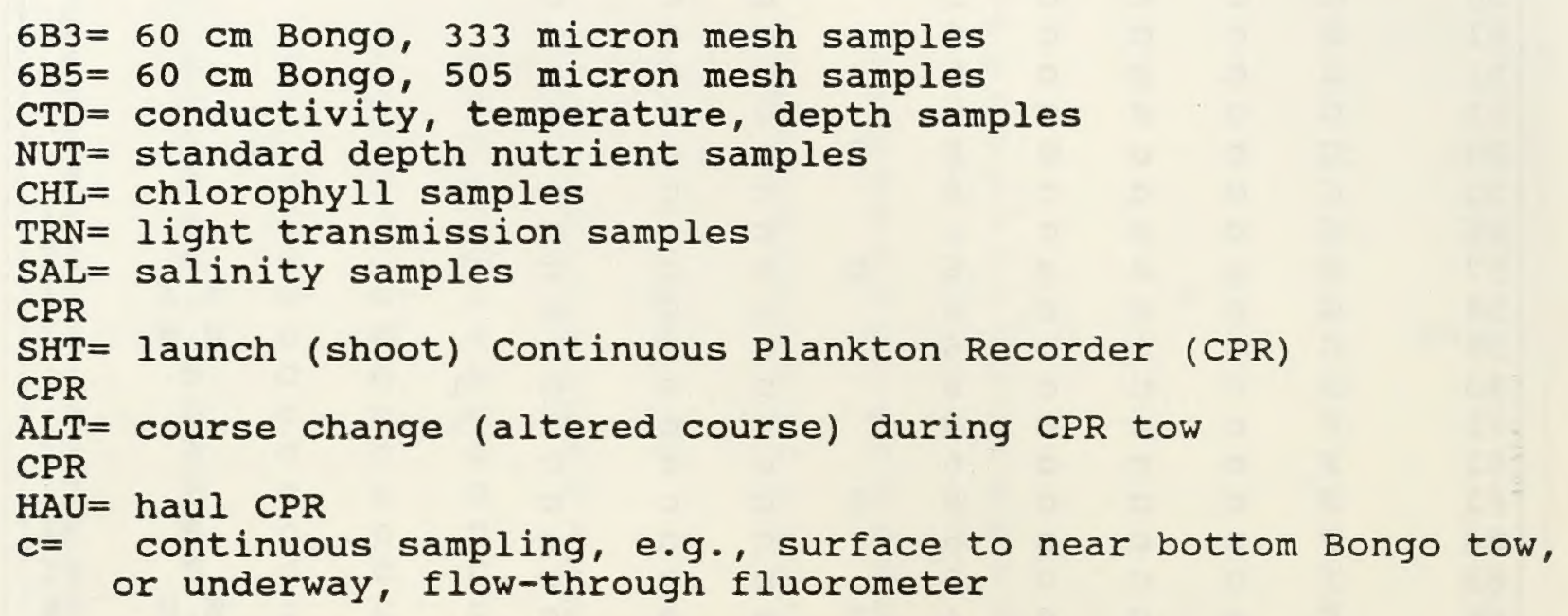




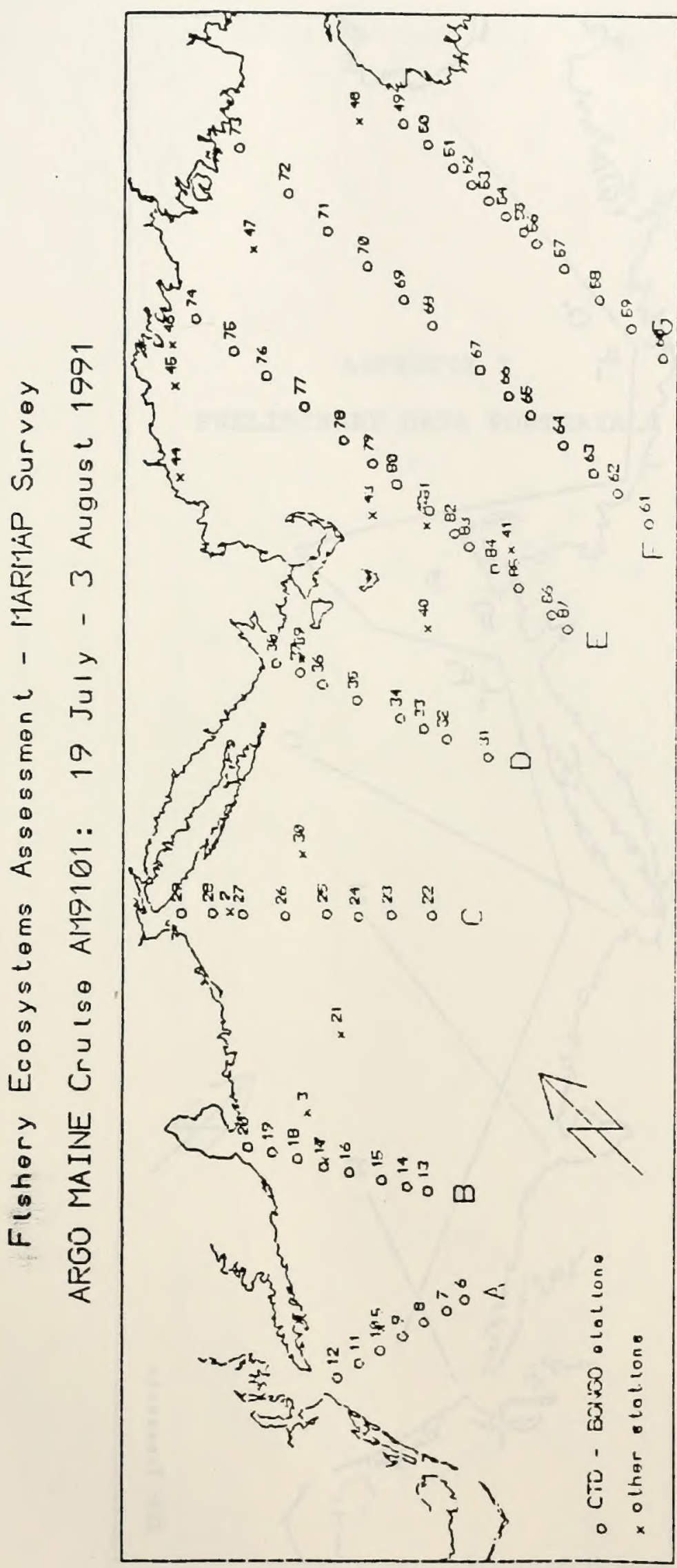

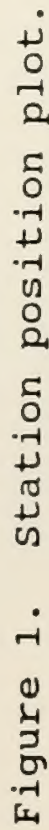




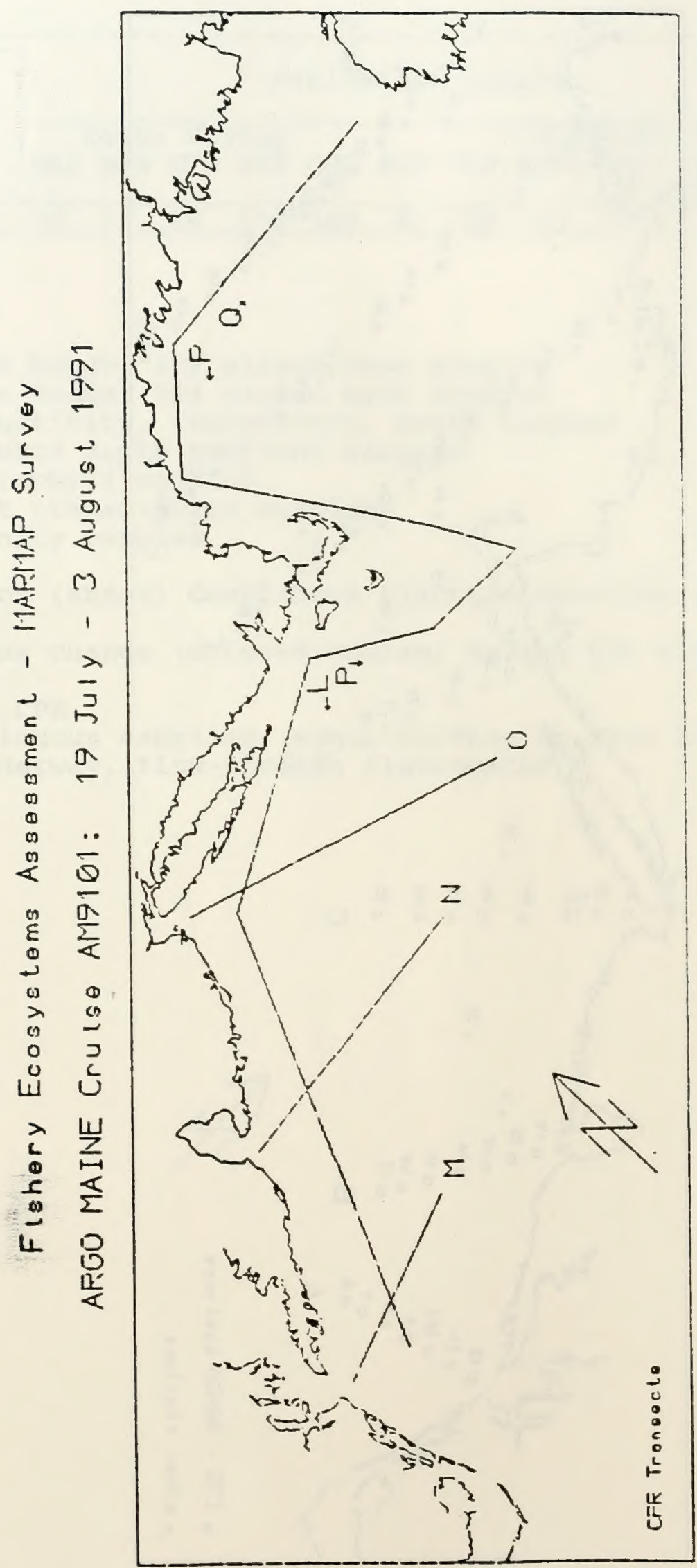

ن 
APPENDIX I

PRELIMINARY DATA PORTRAYALS 


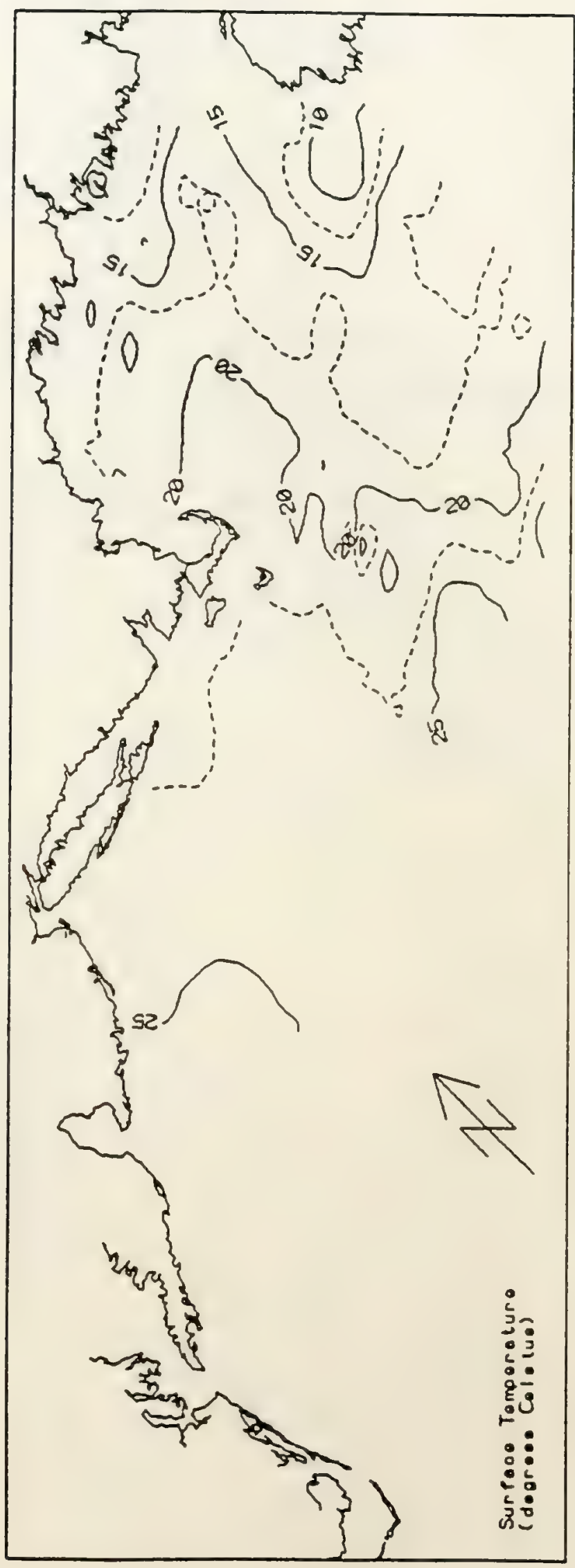




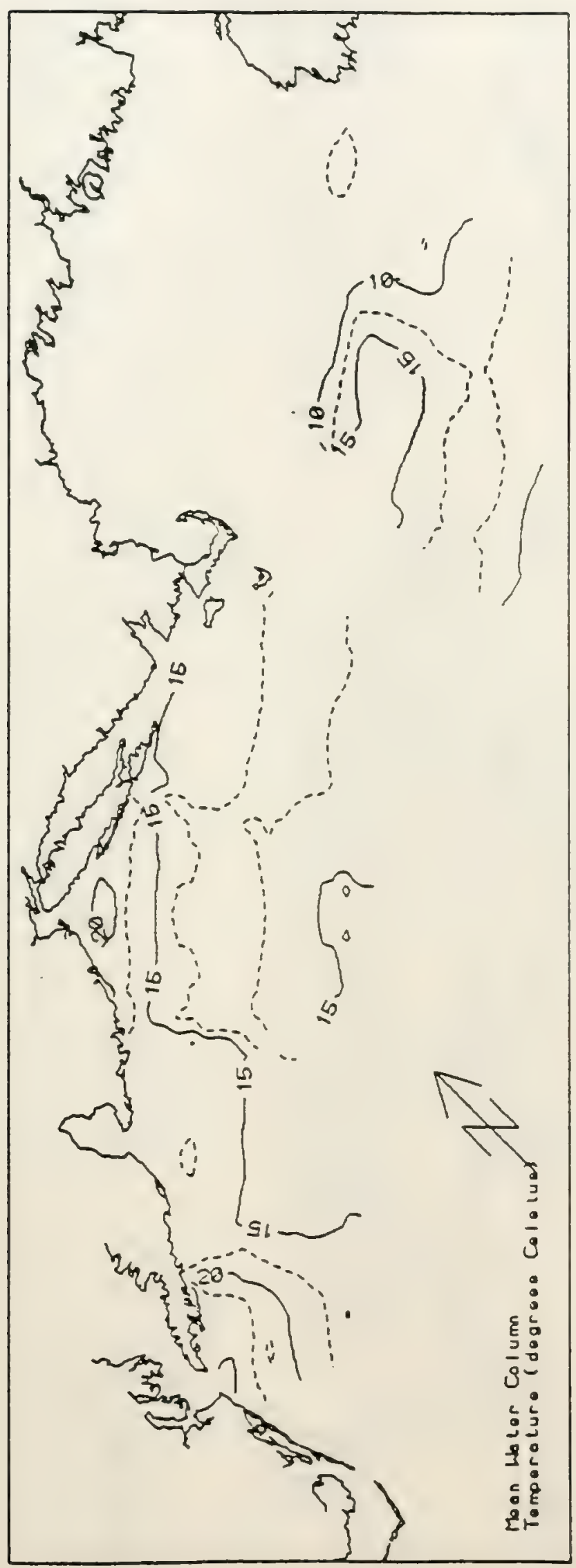




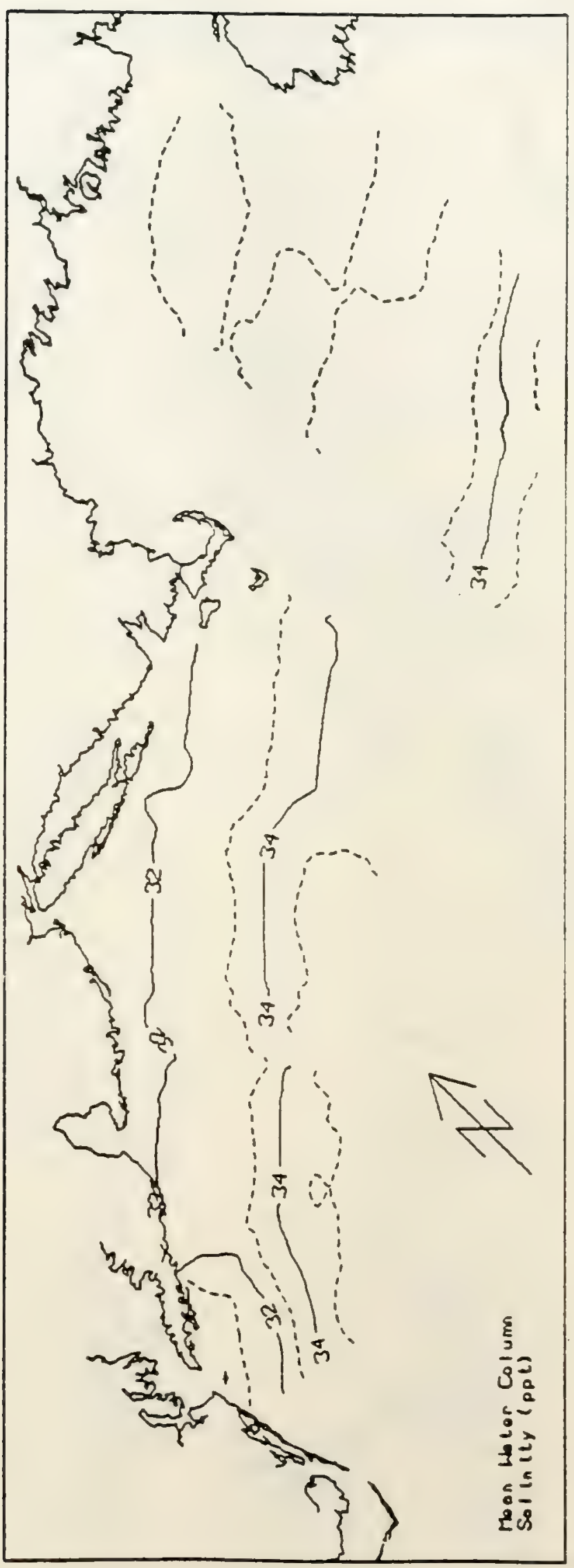




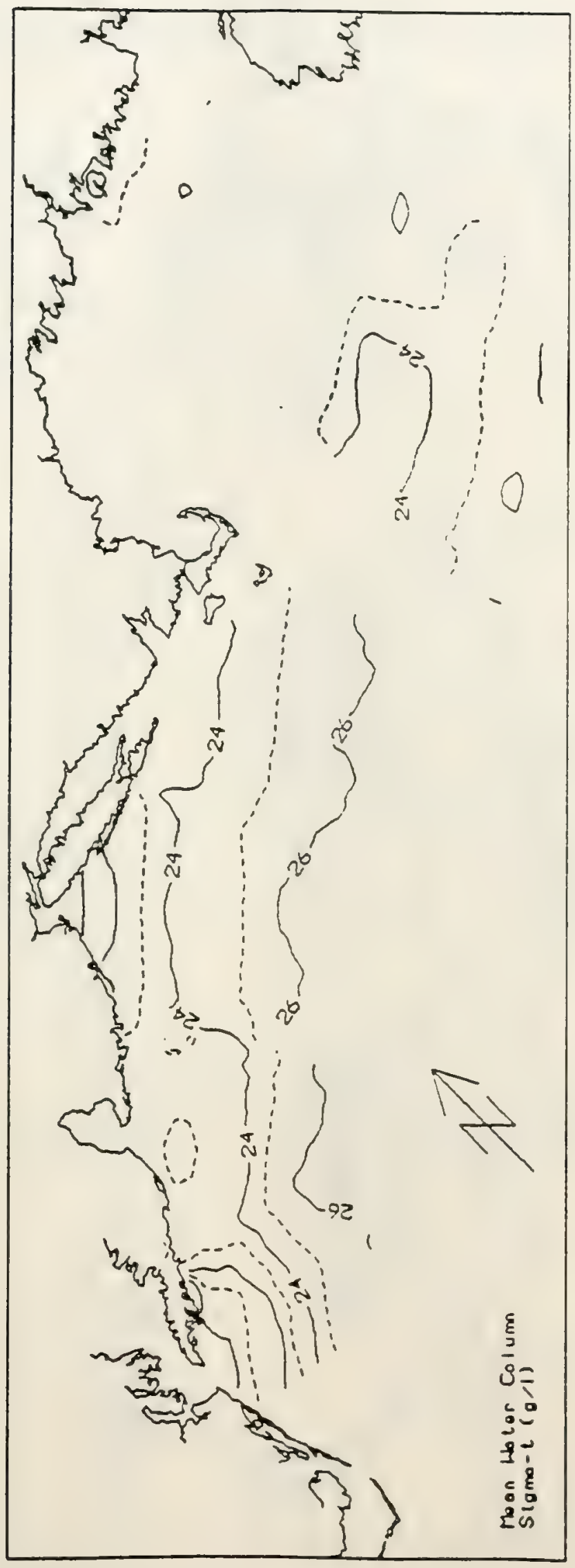




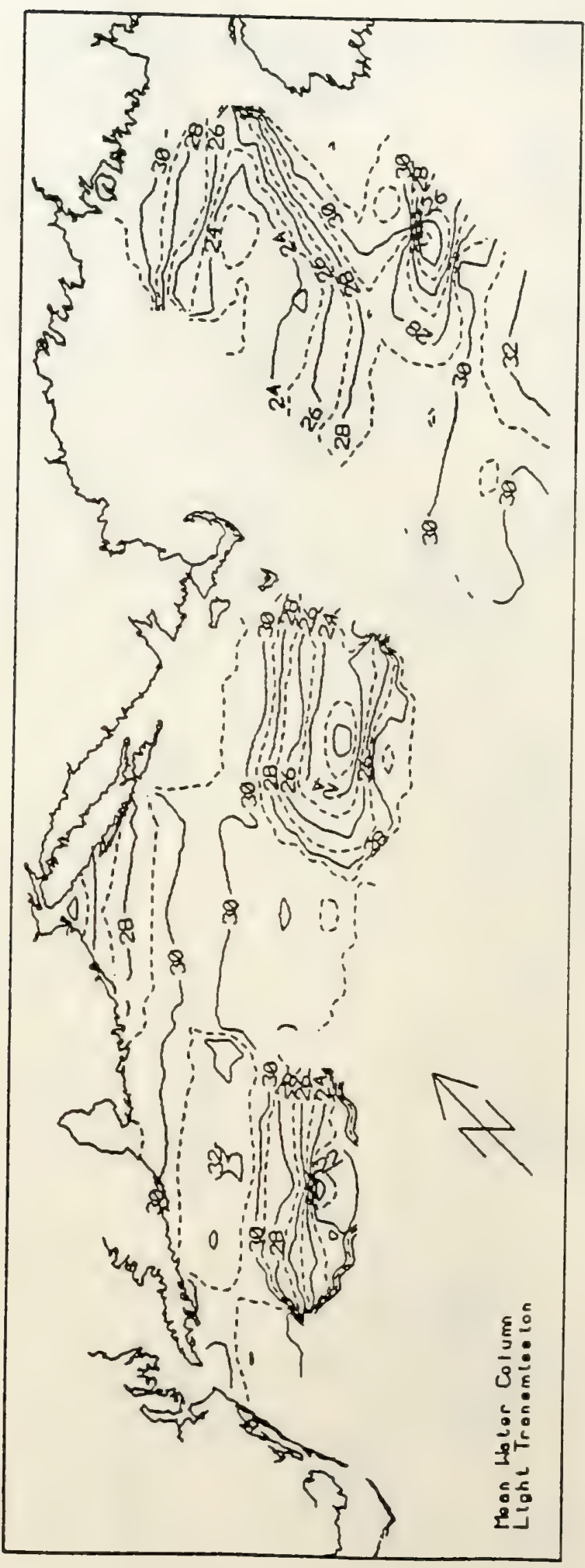




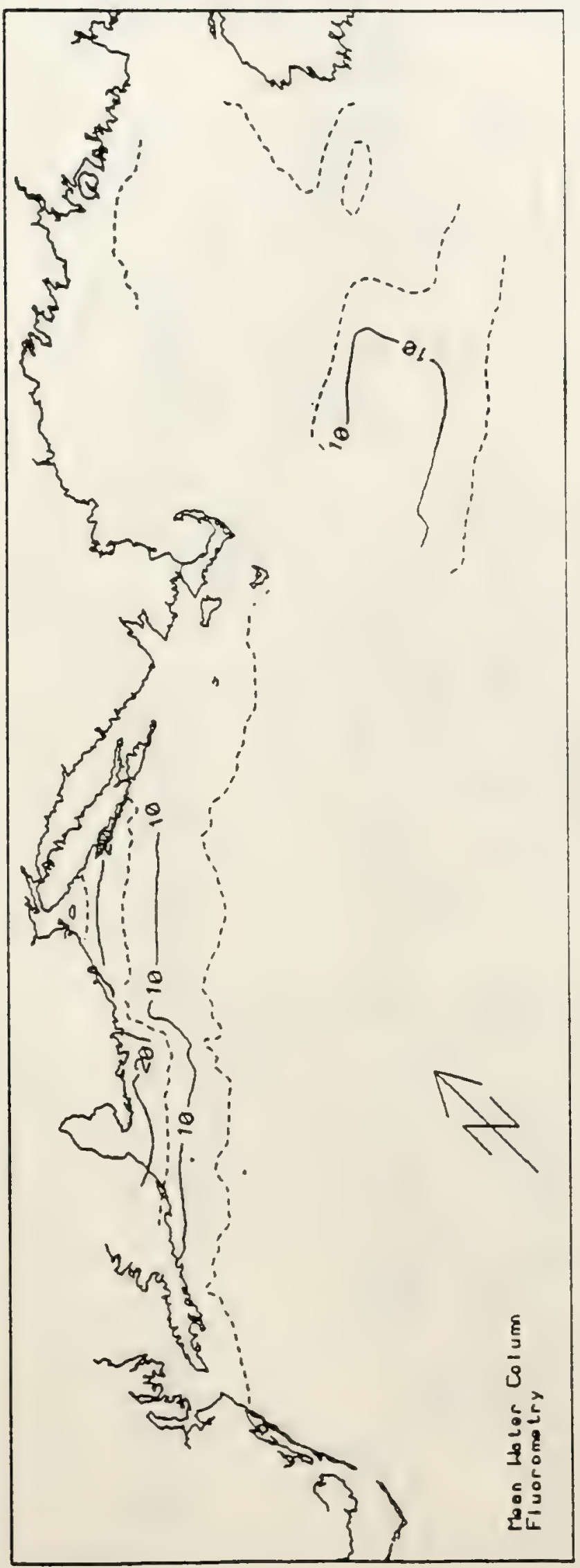




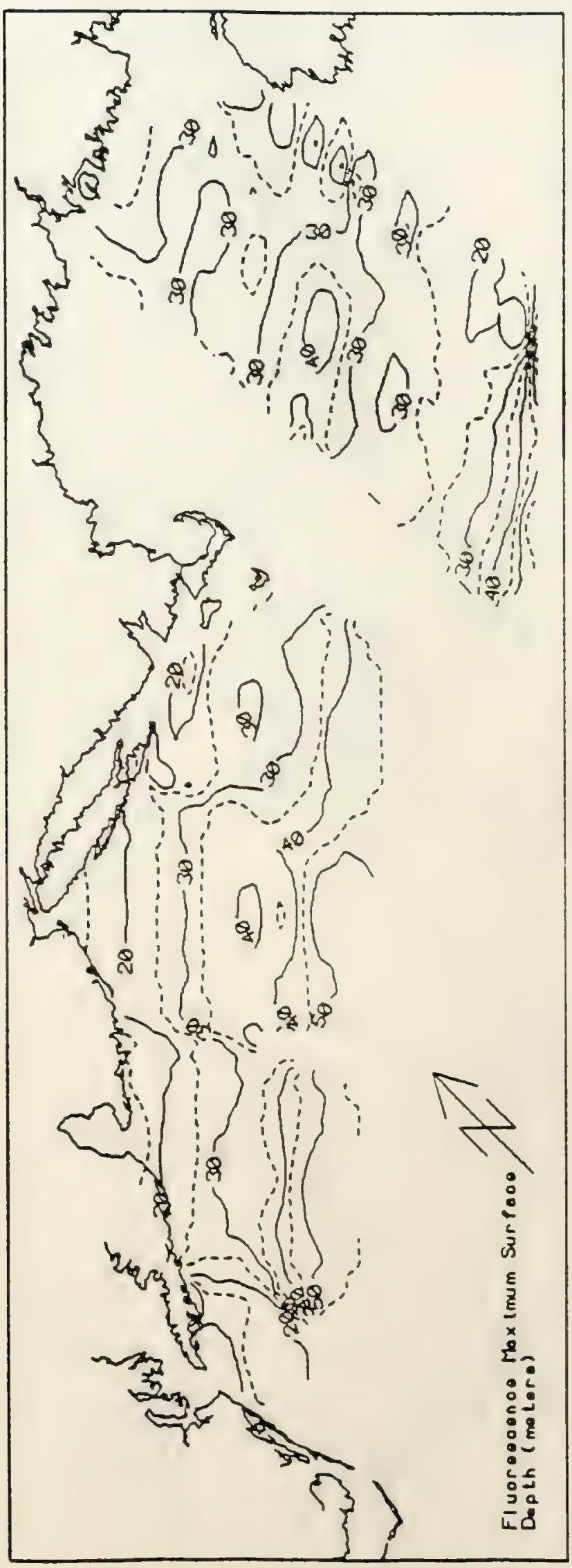




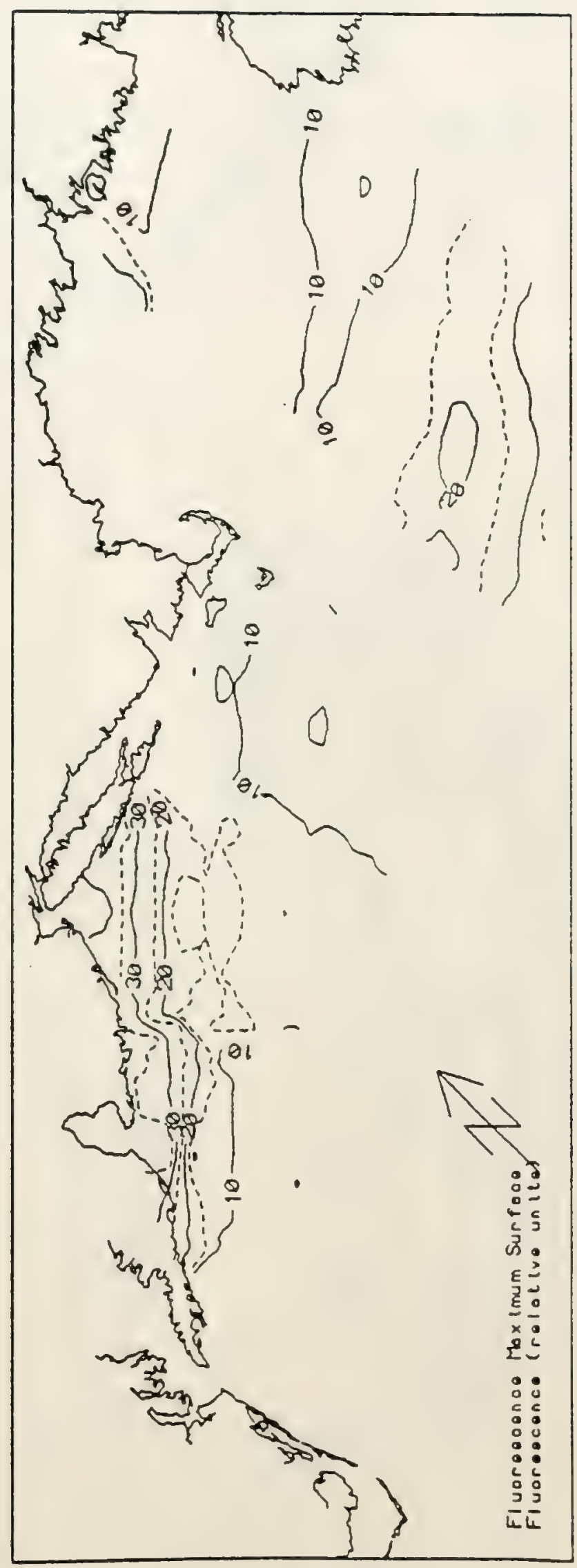




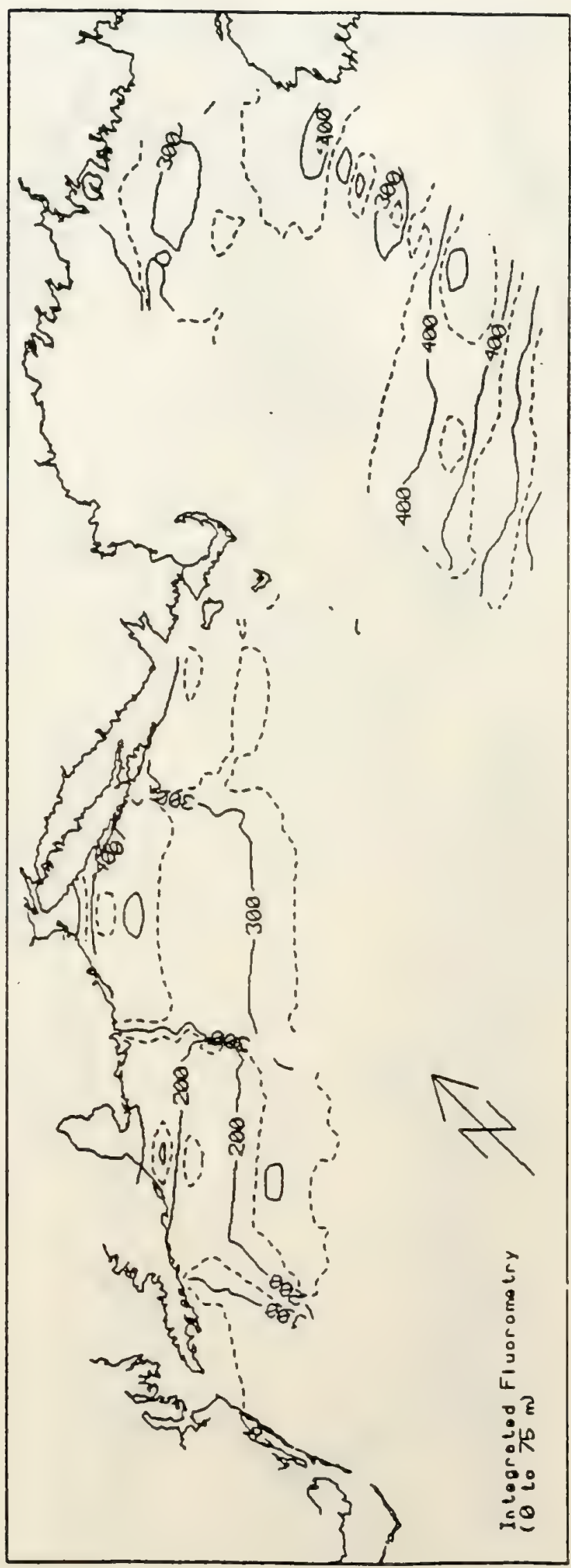




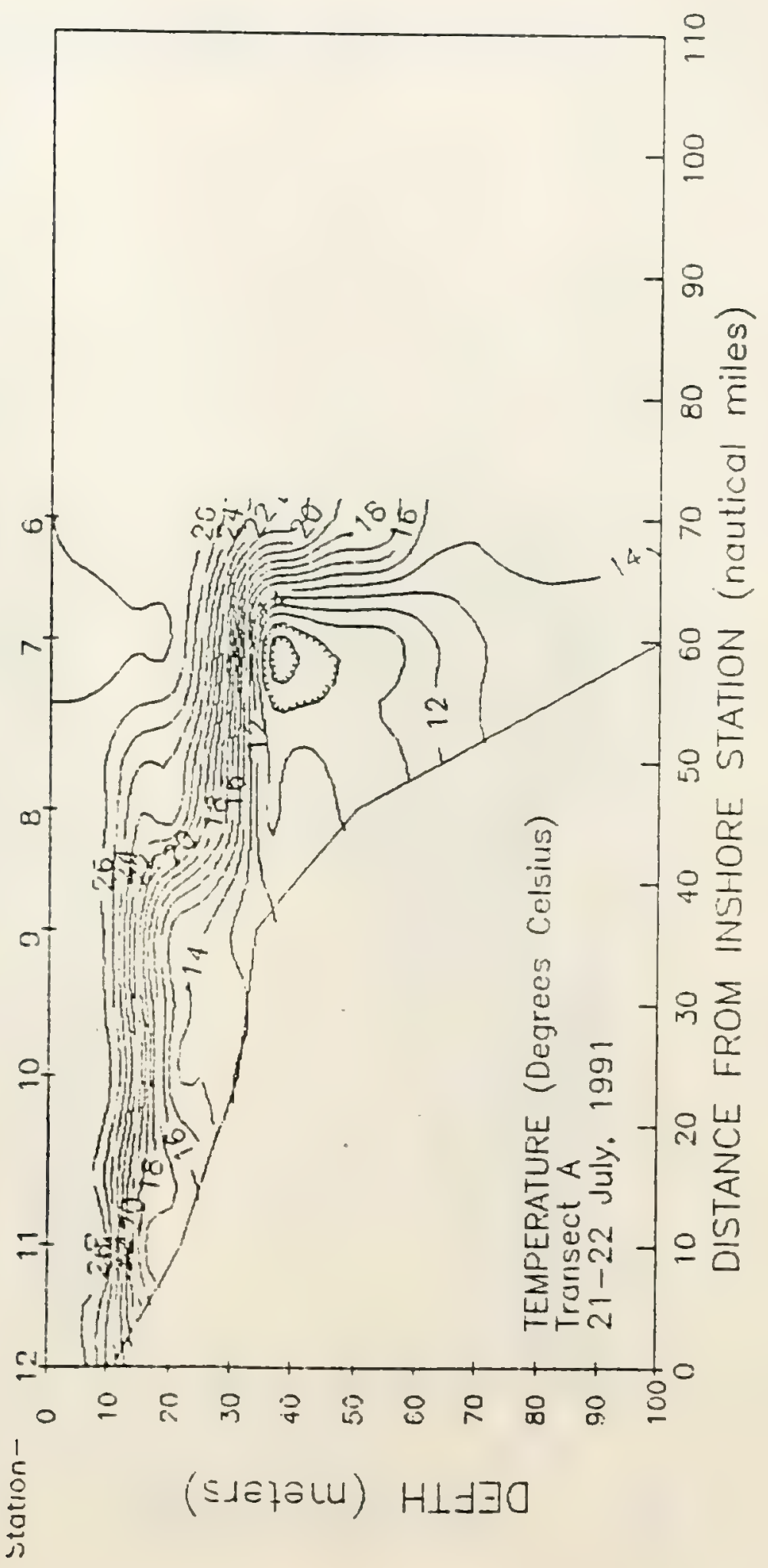




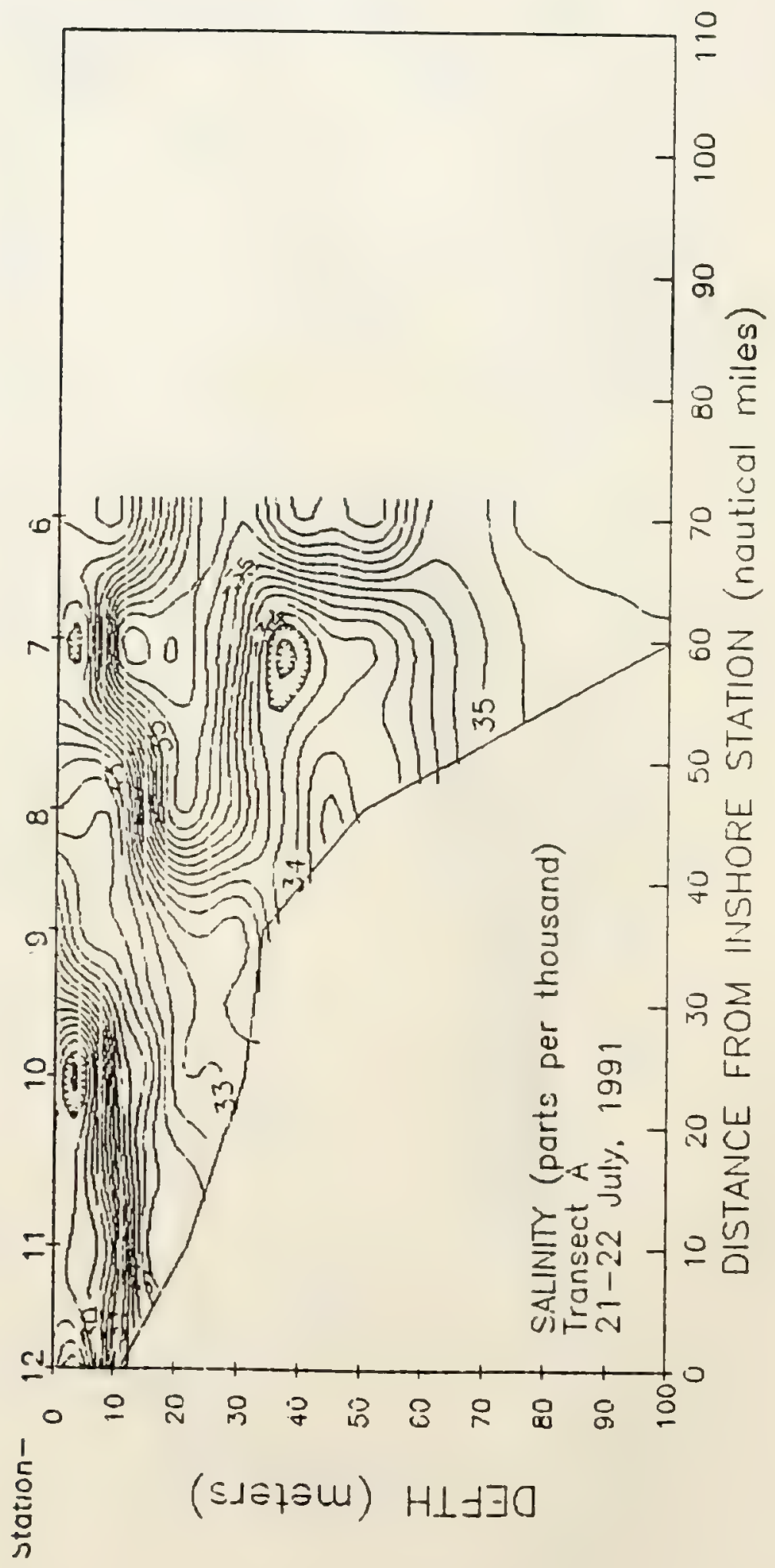




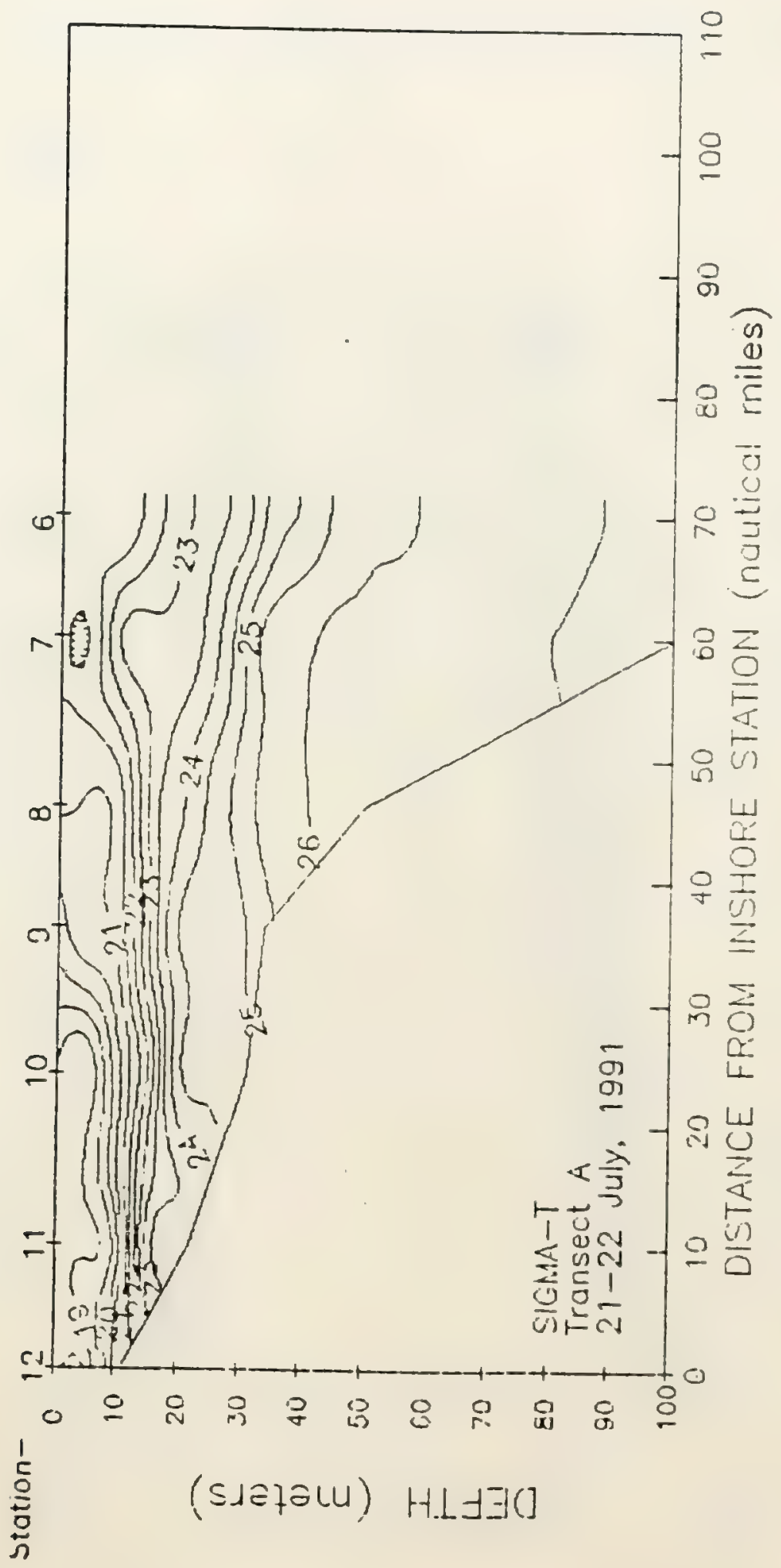




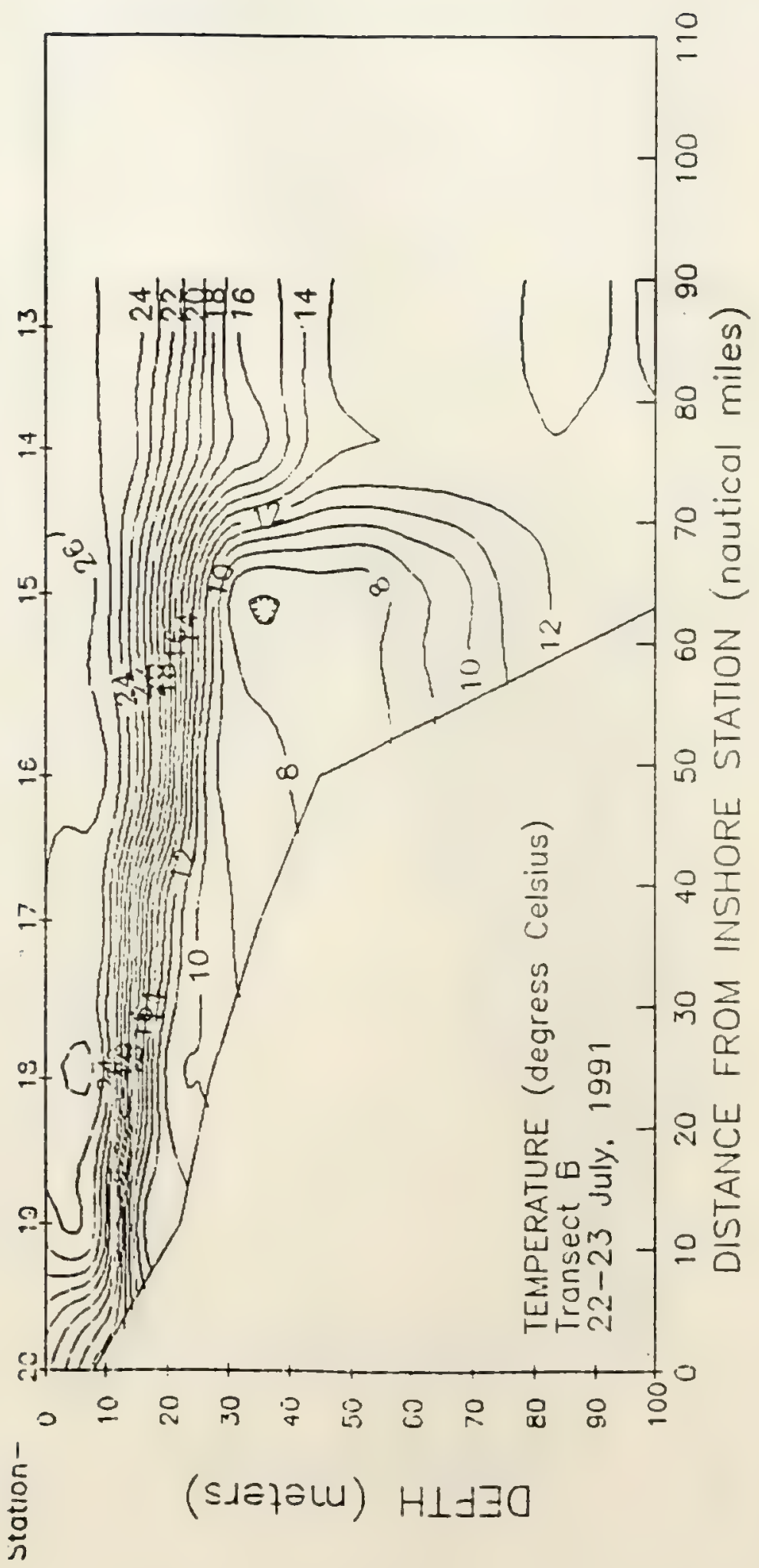




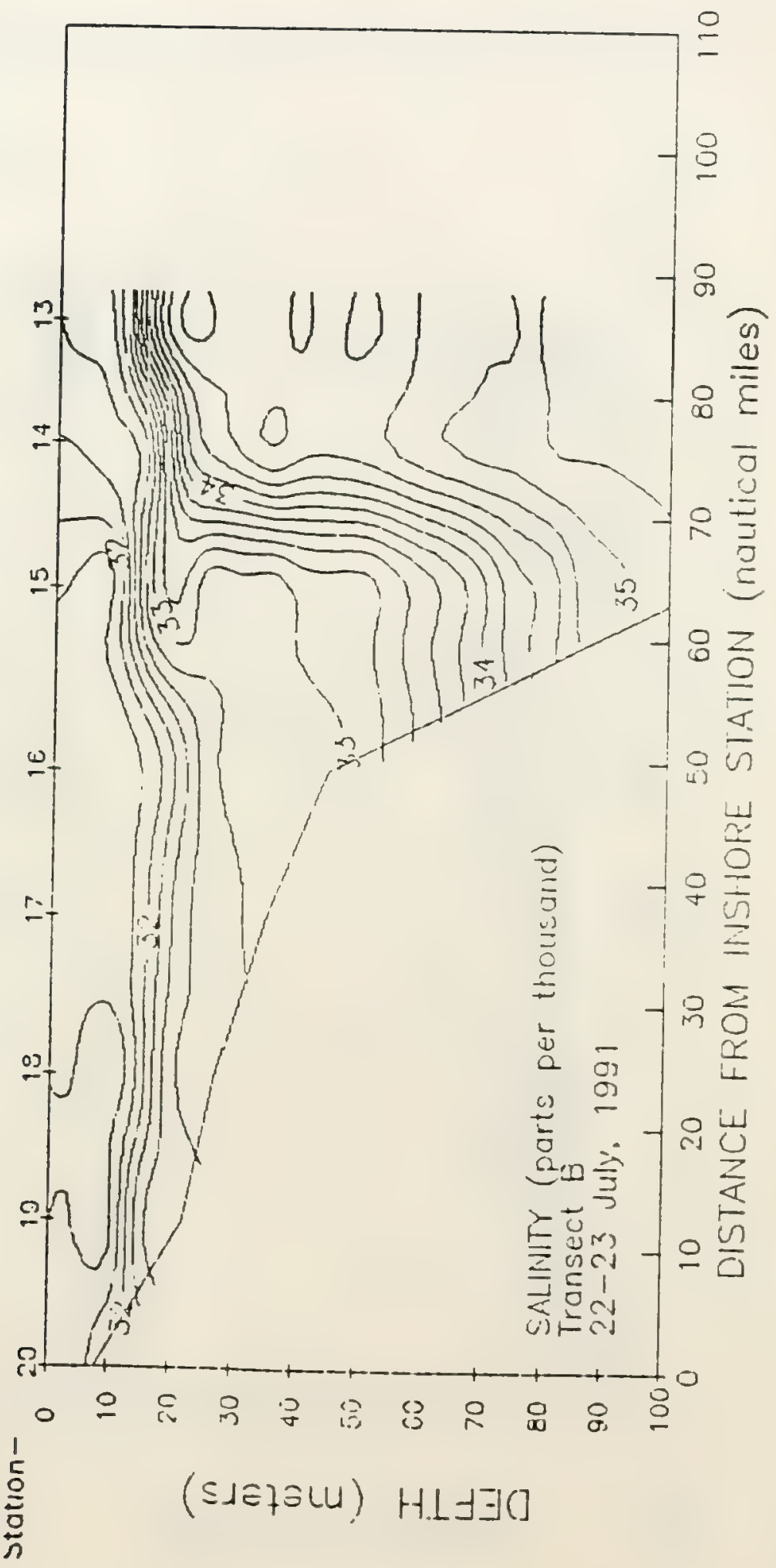




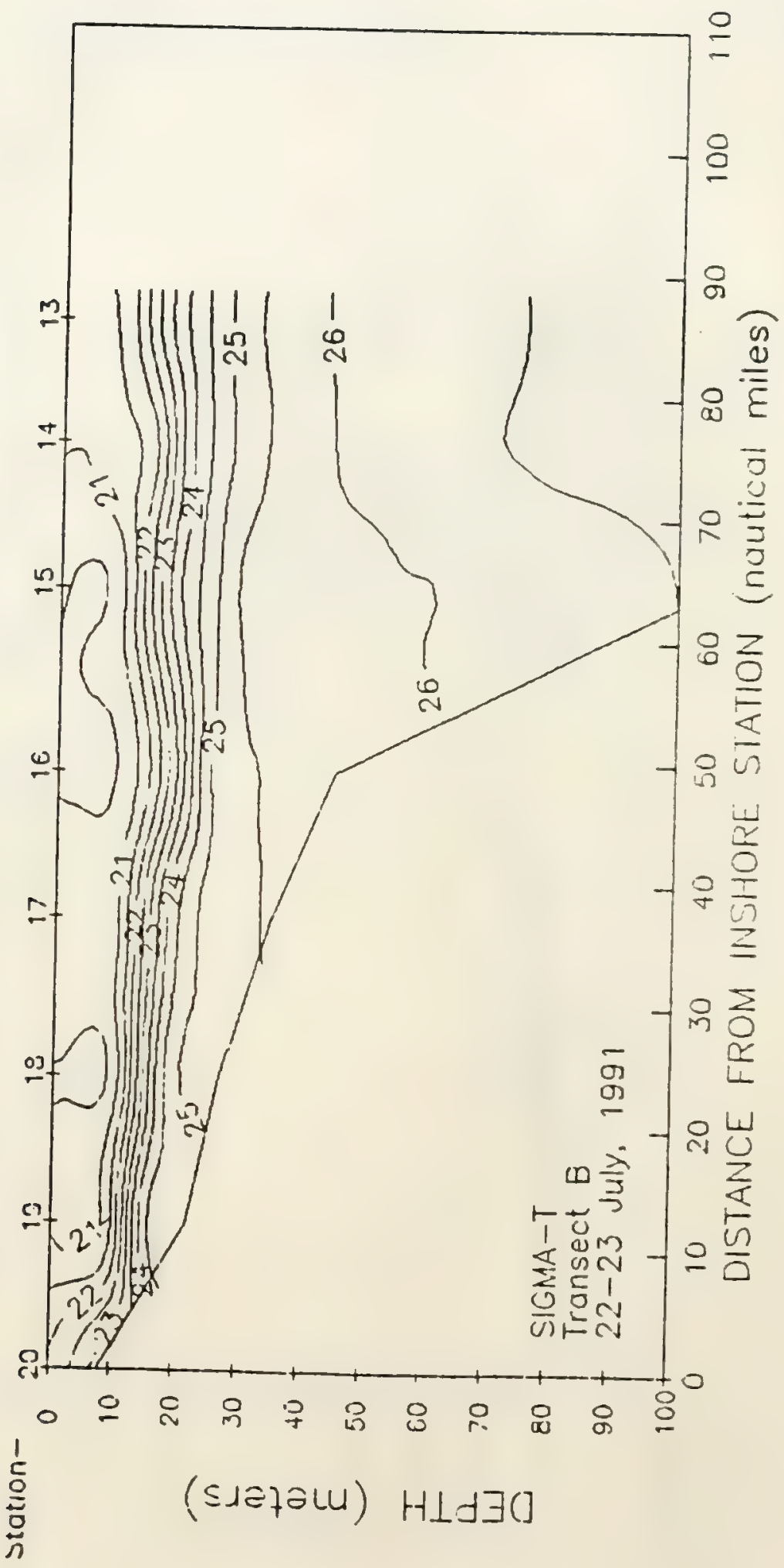




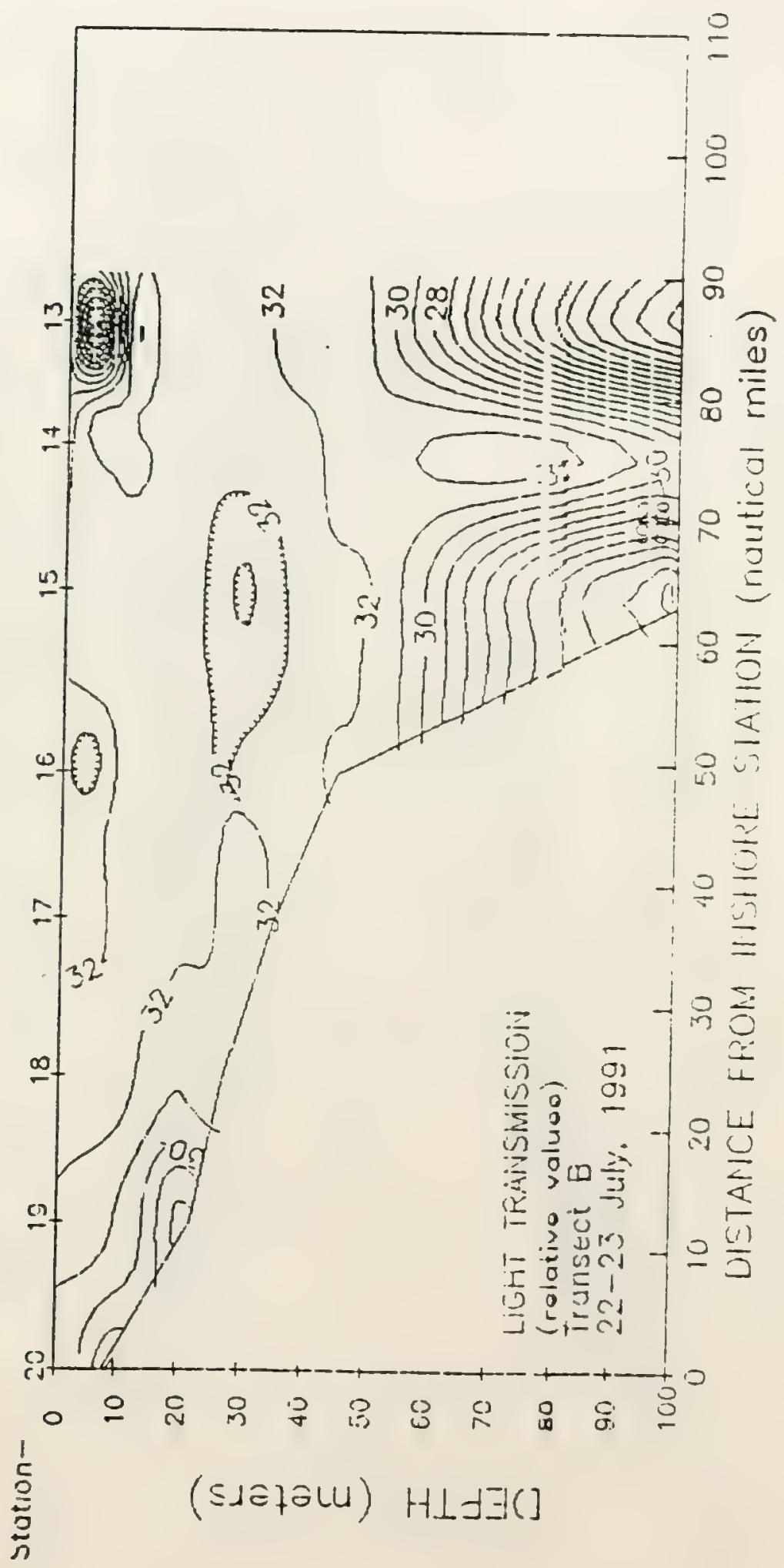




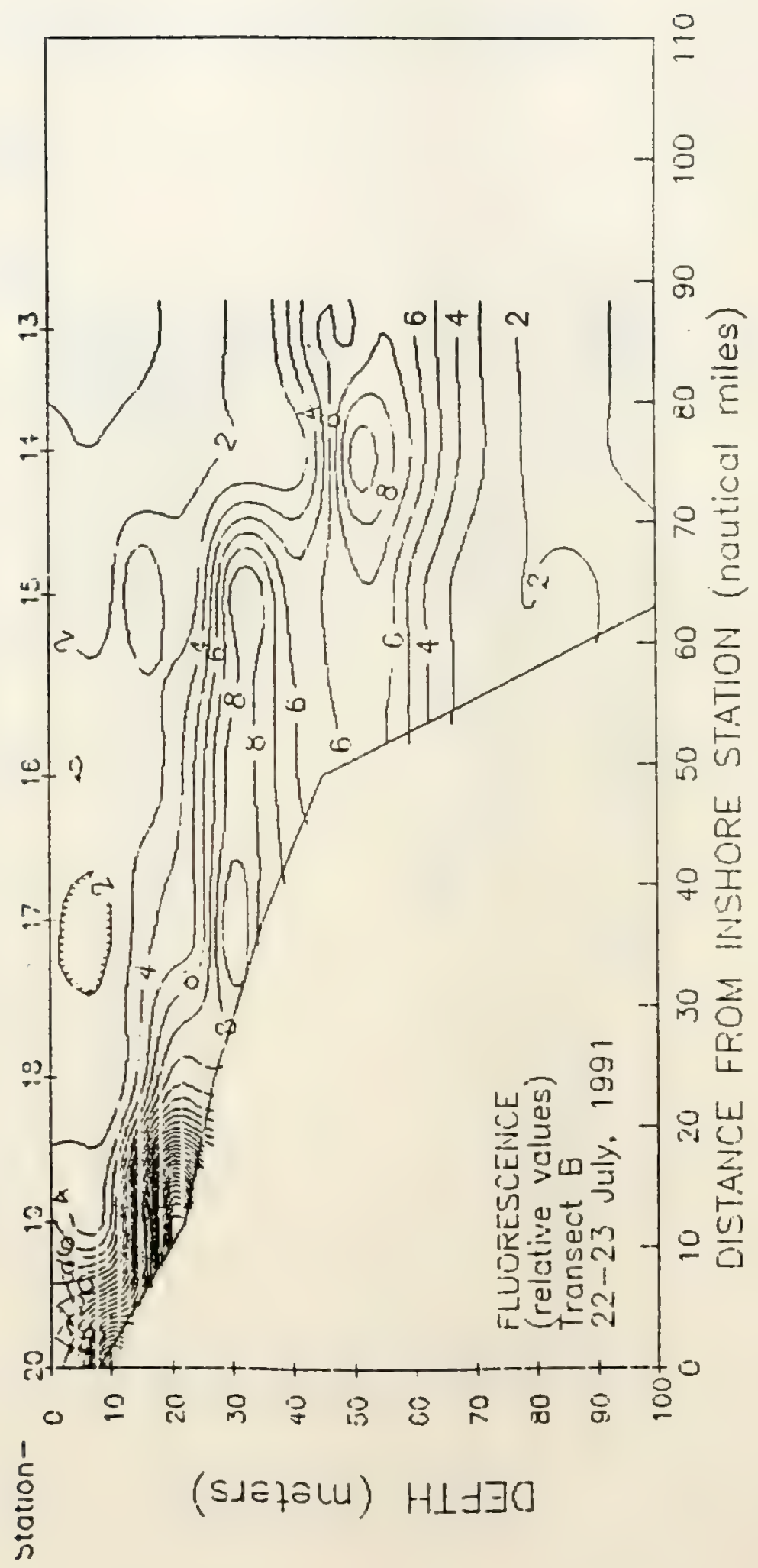




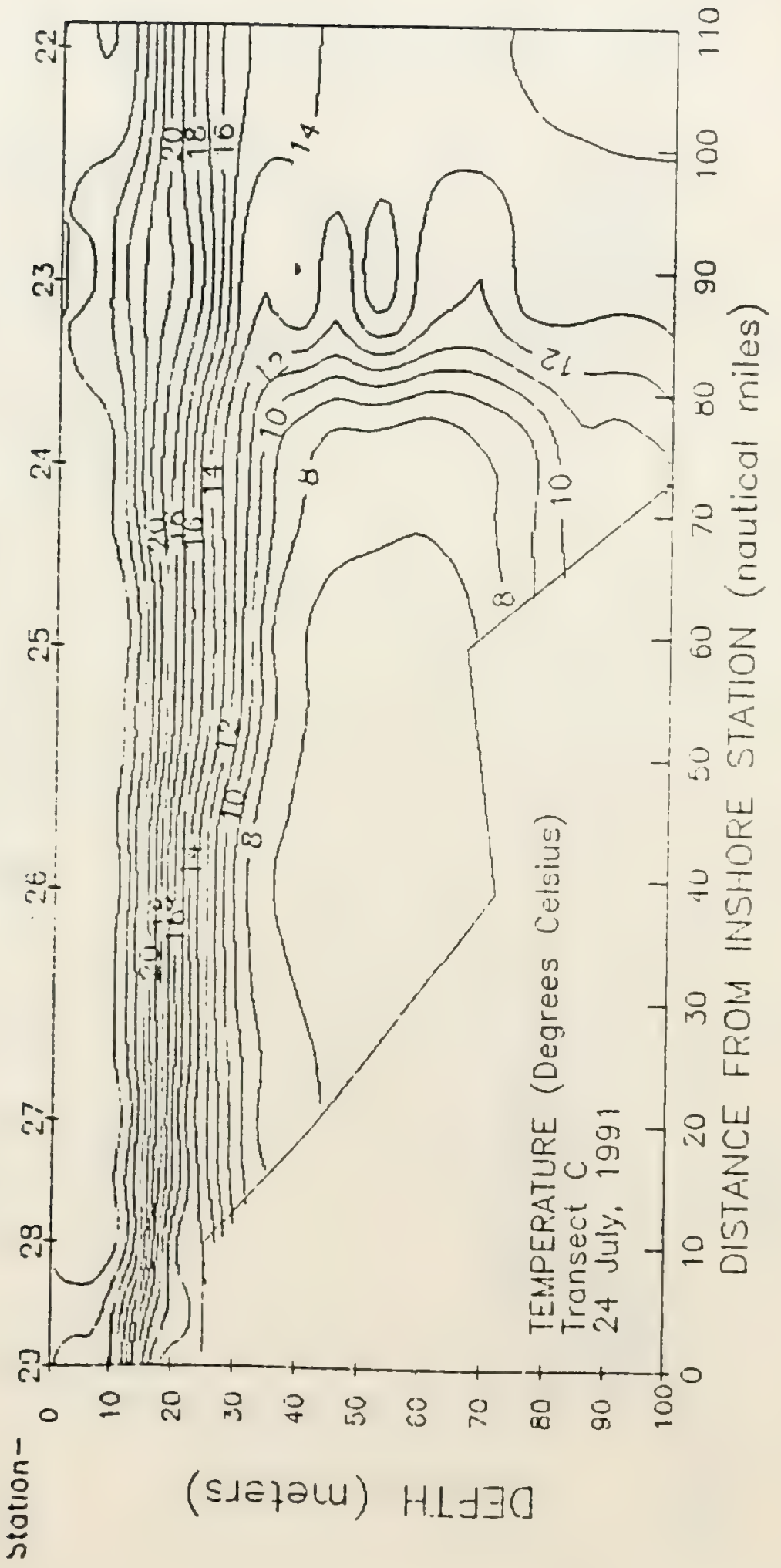




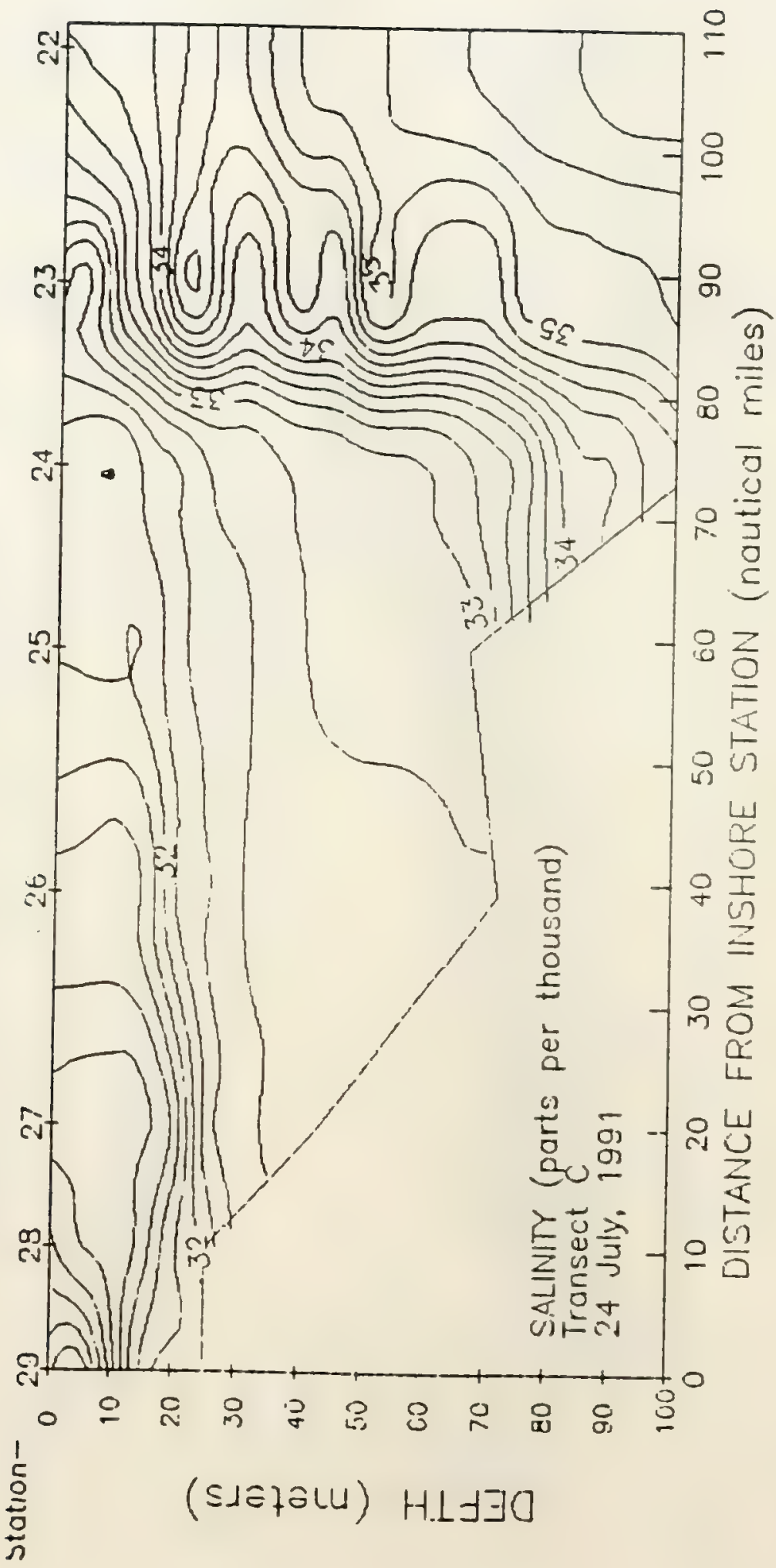




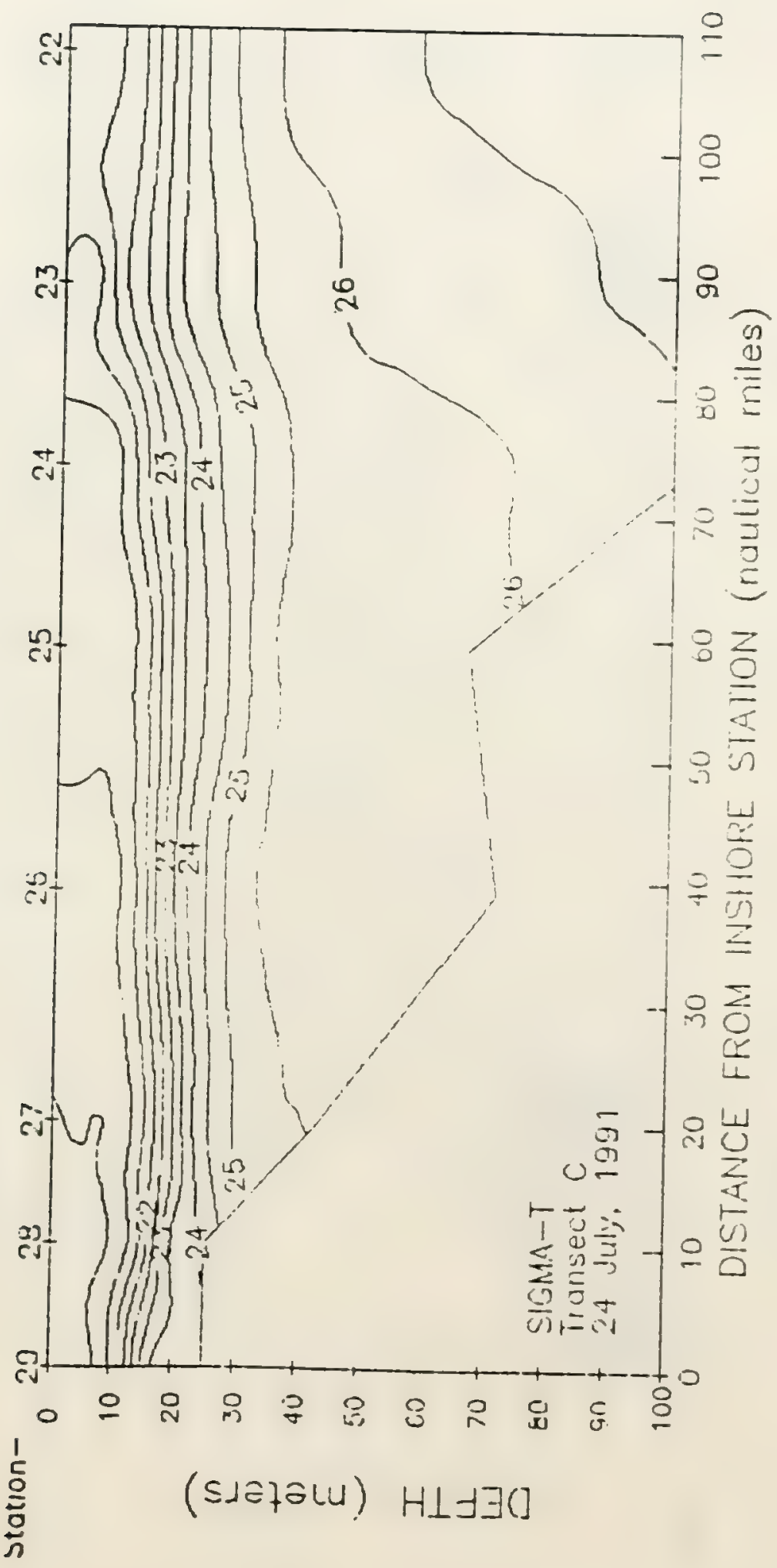




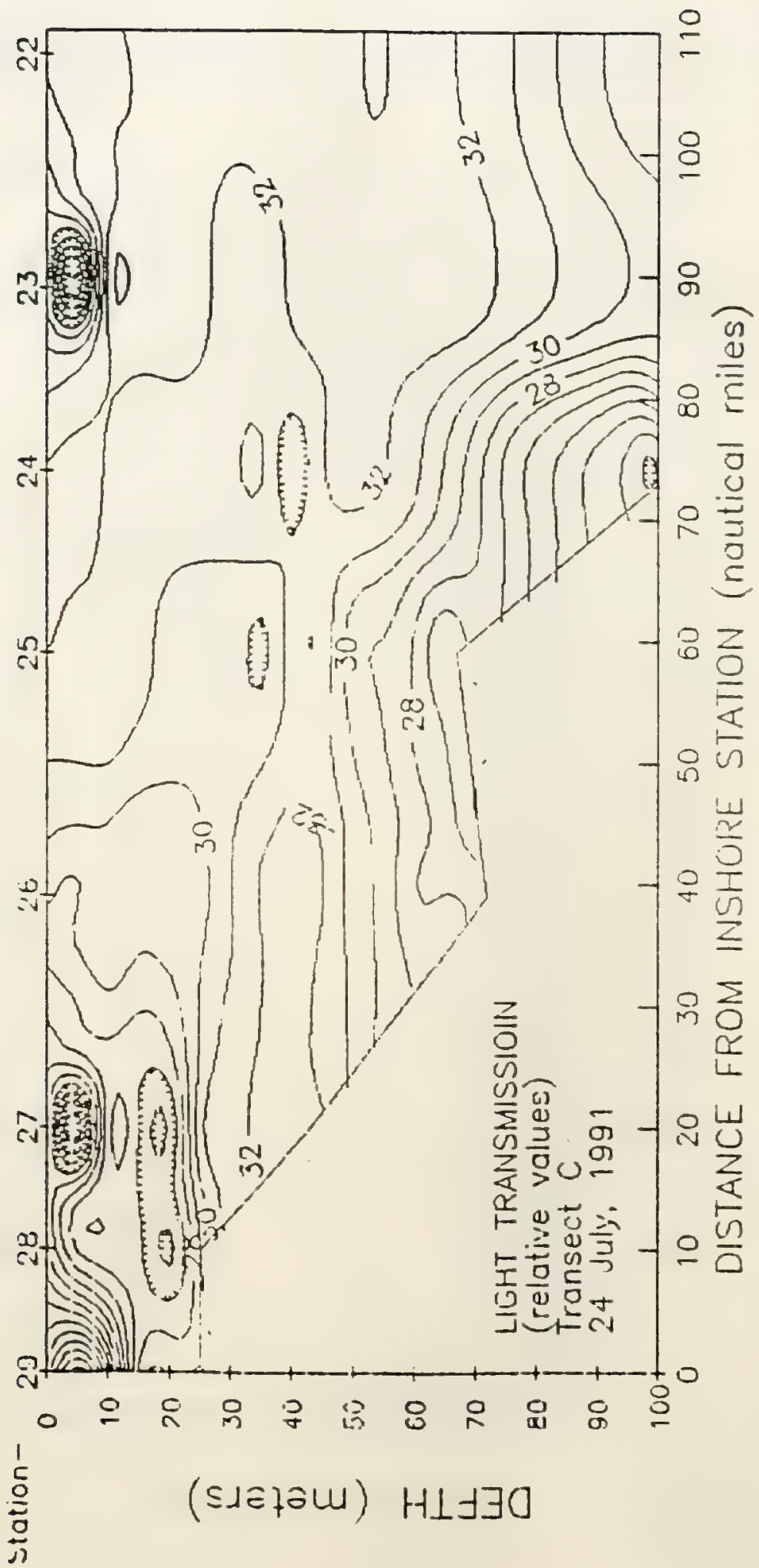




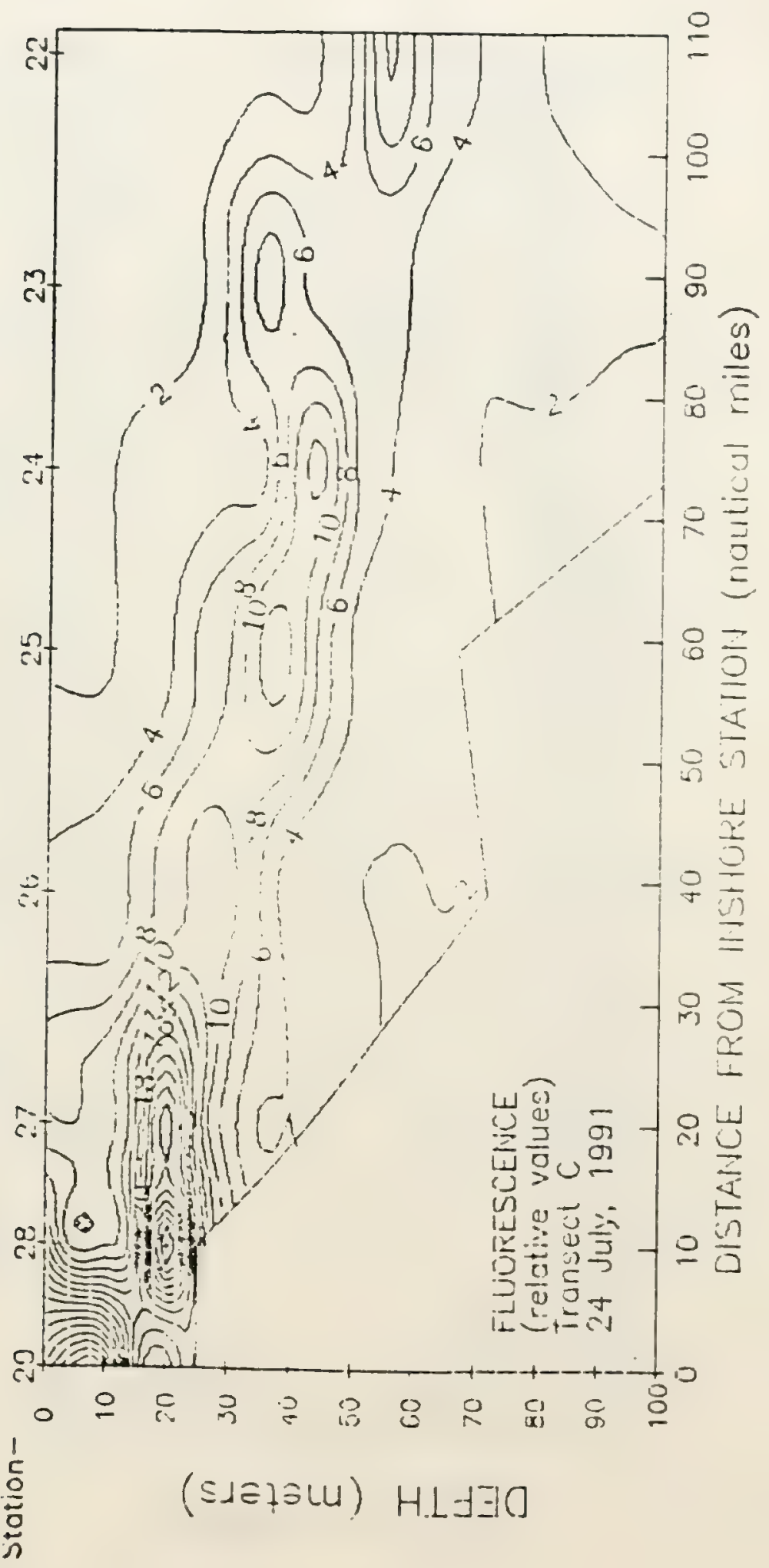




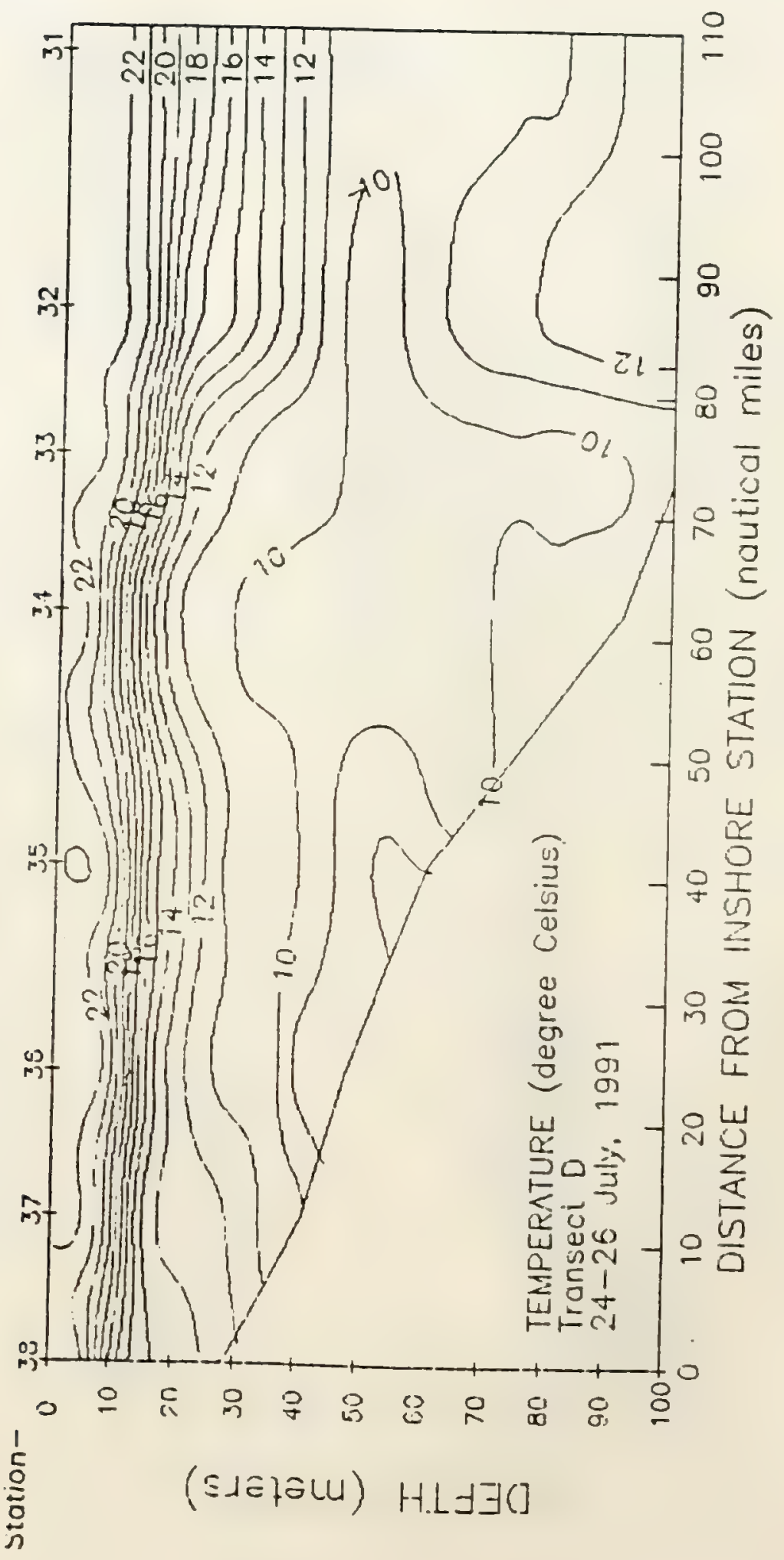




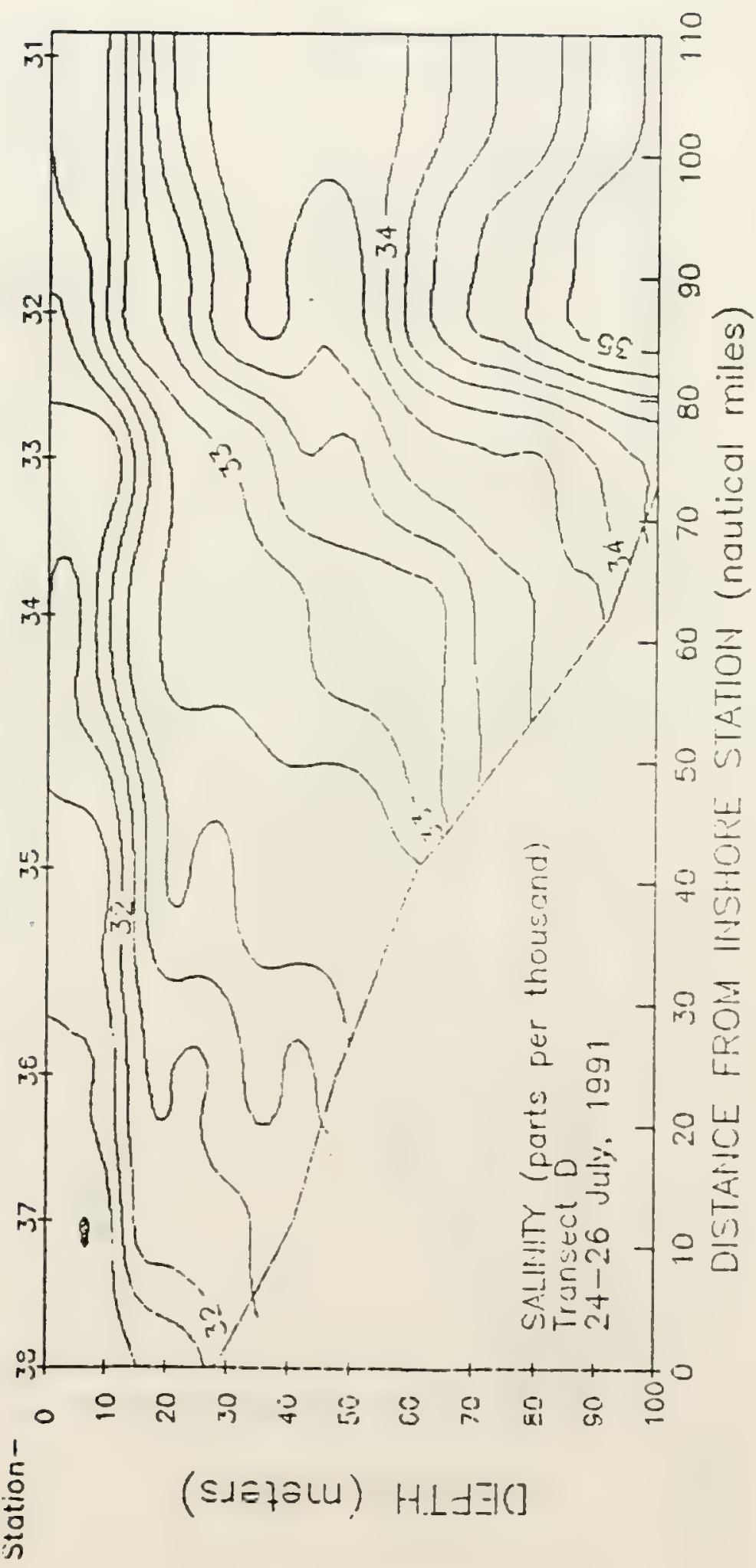




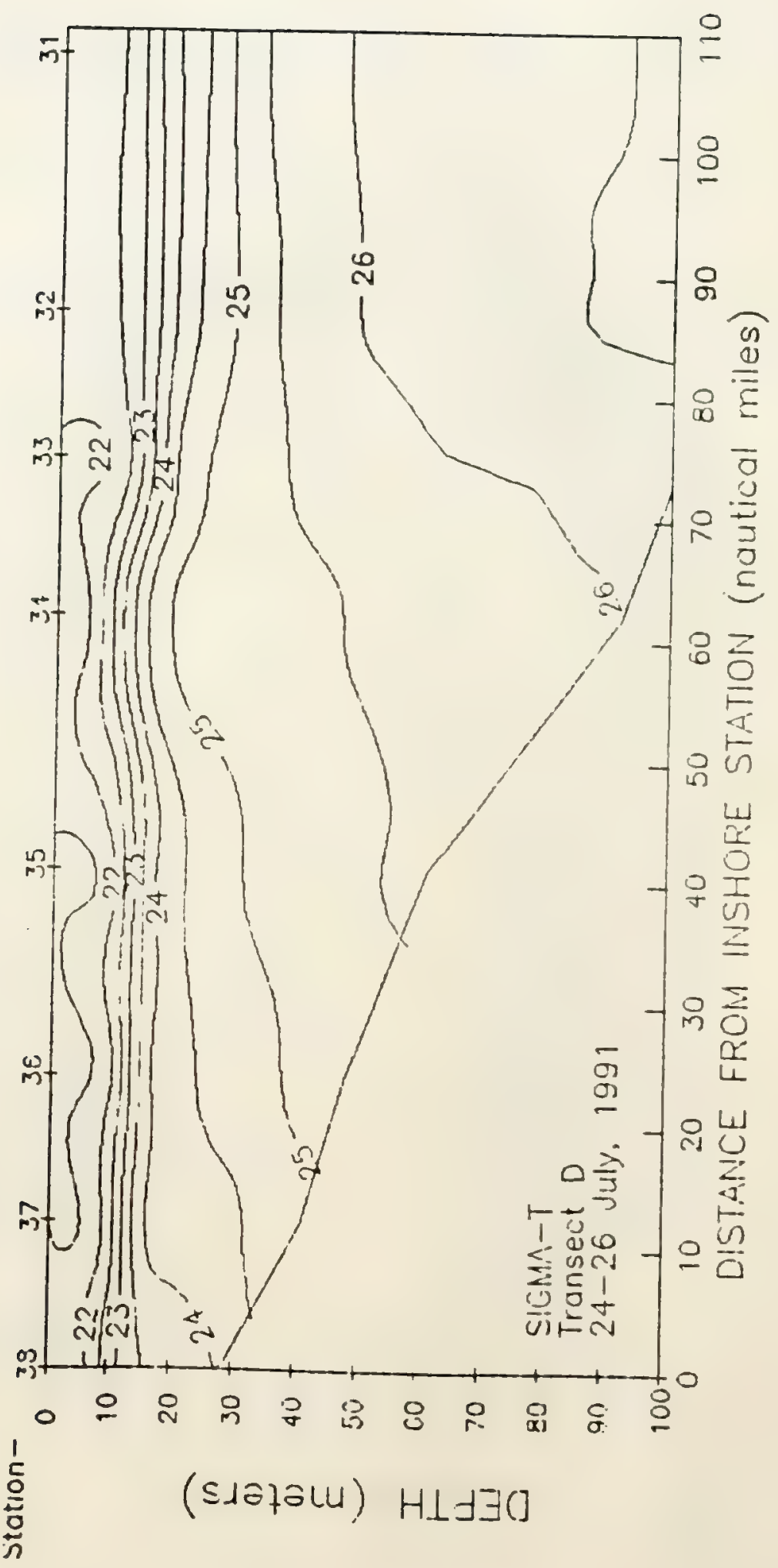




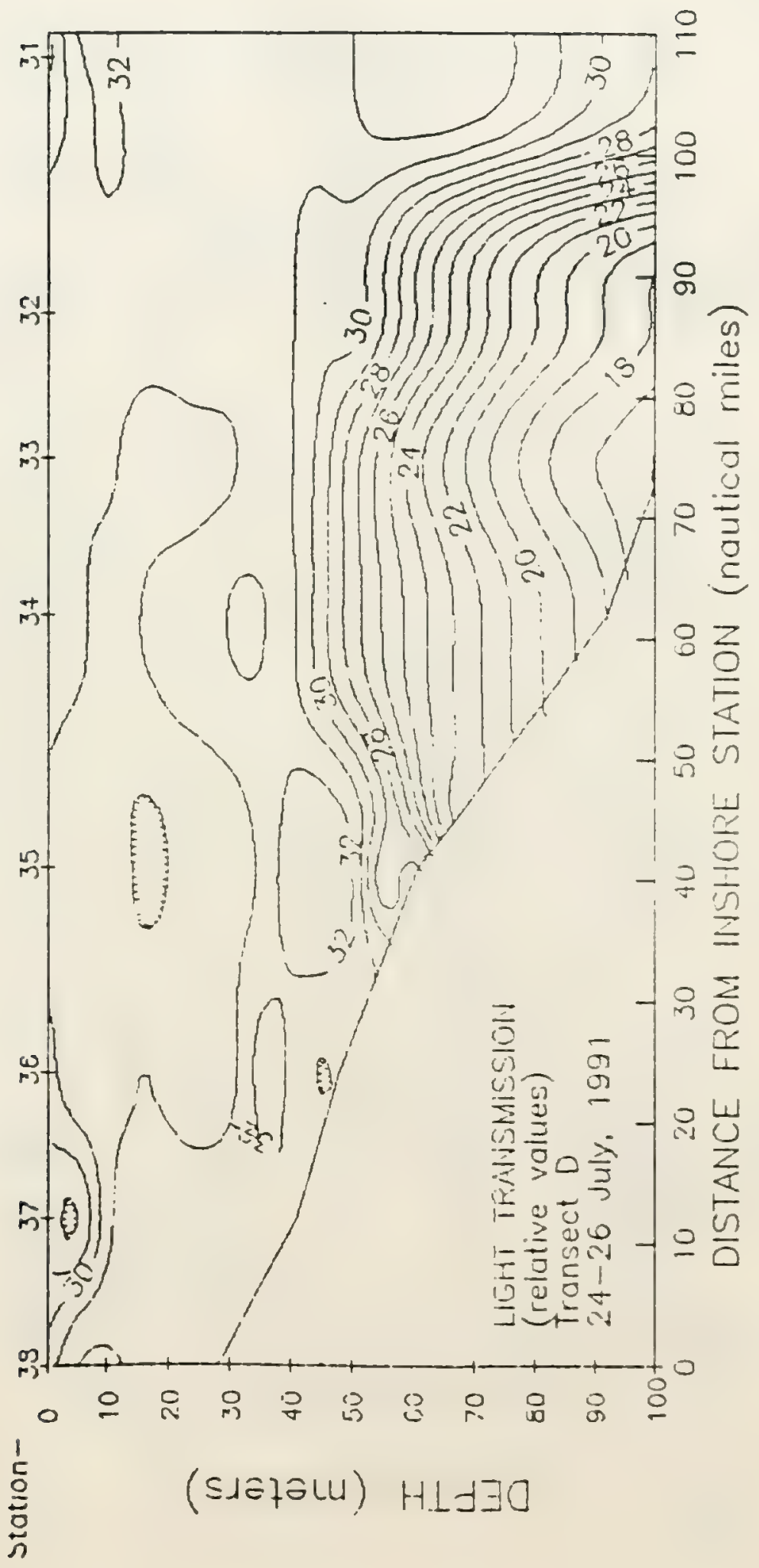




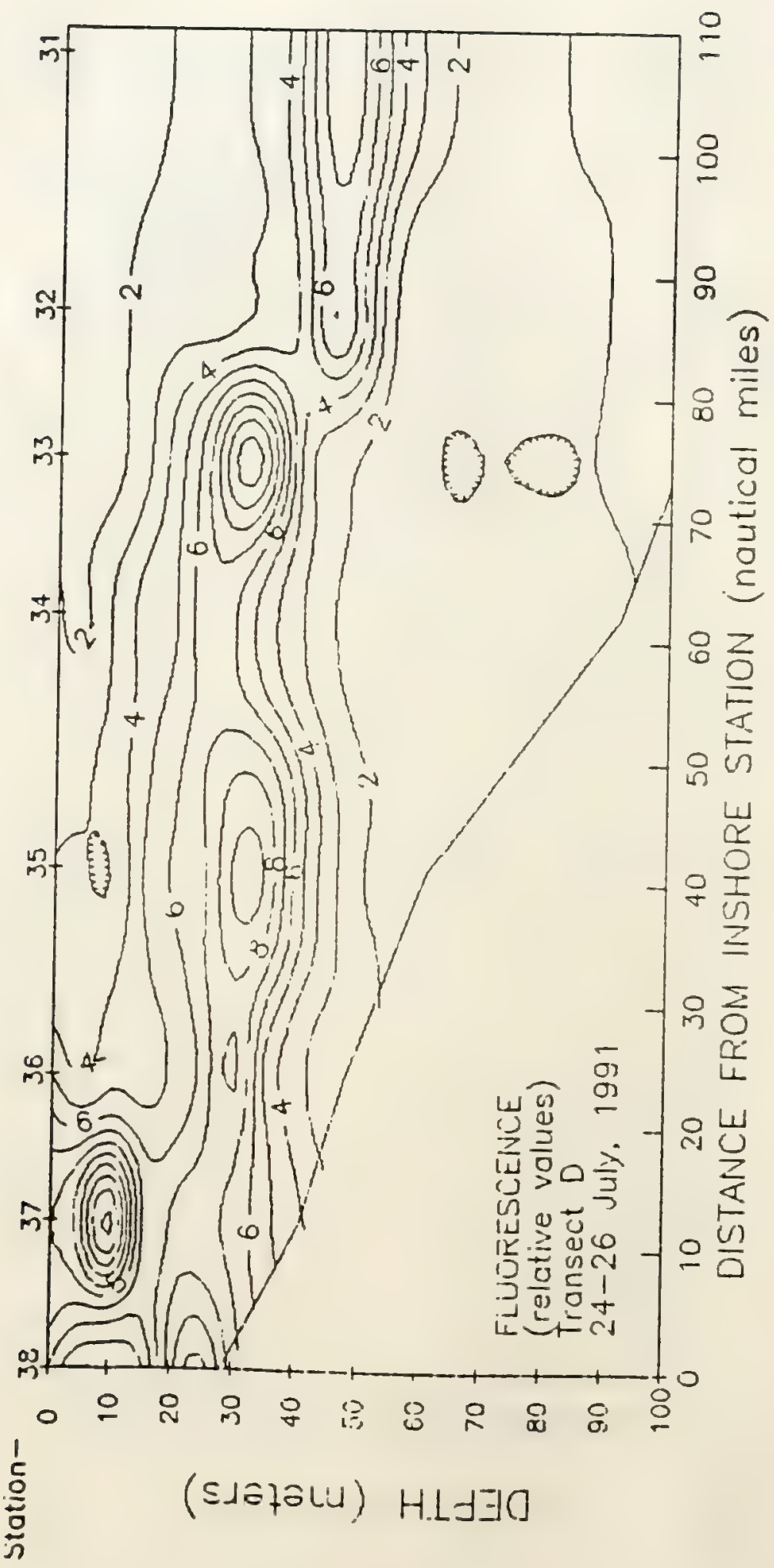




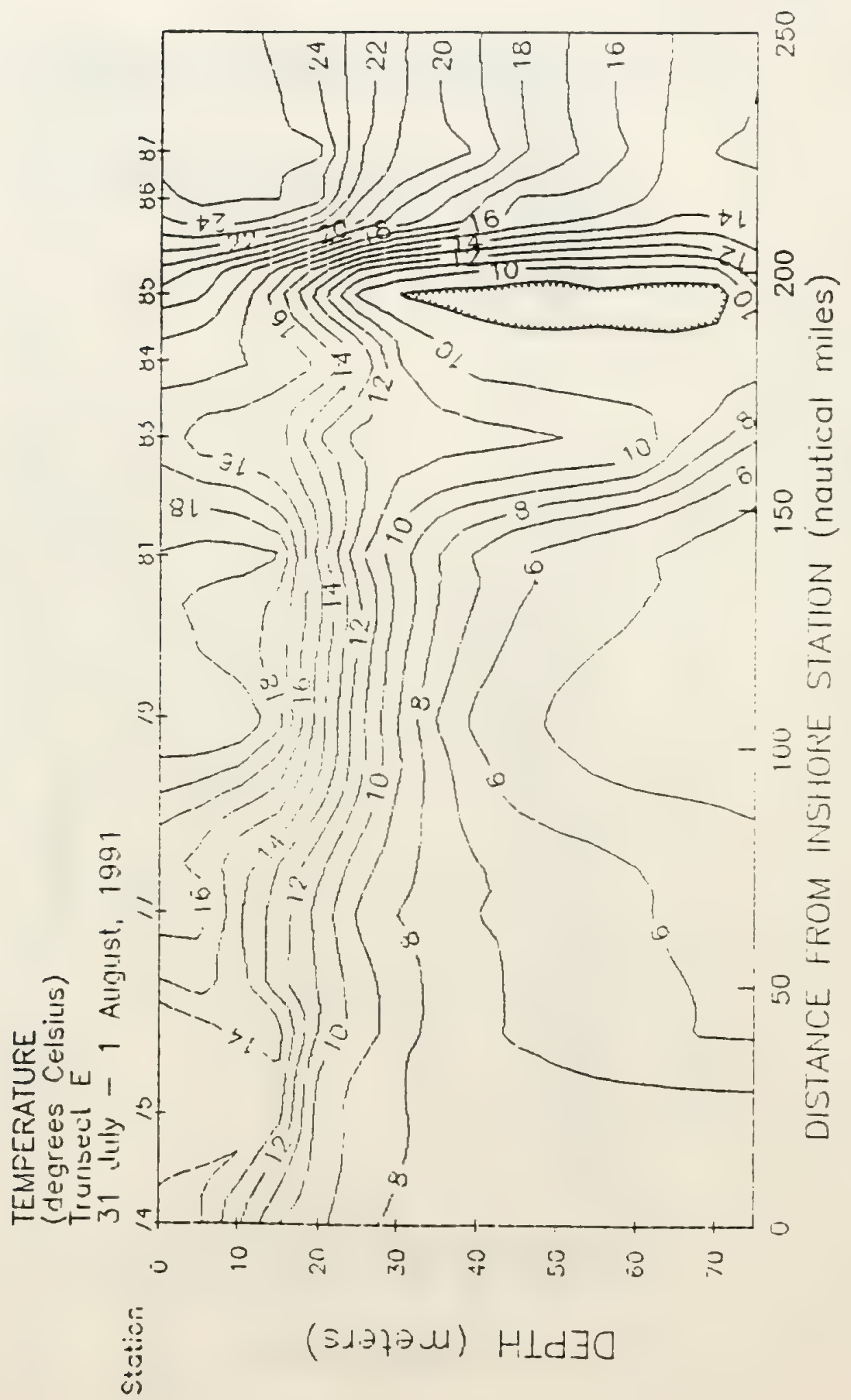




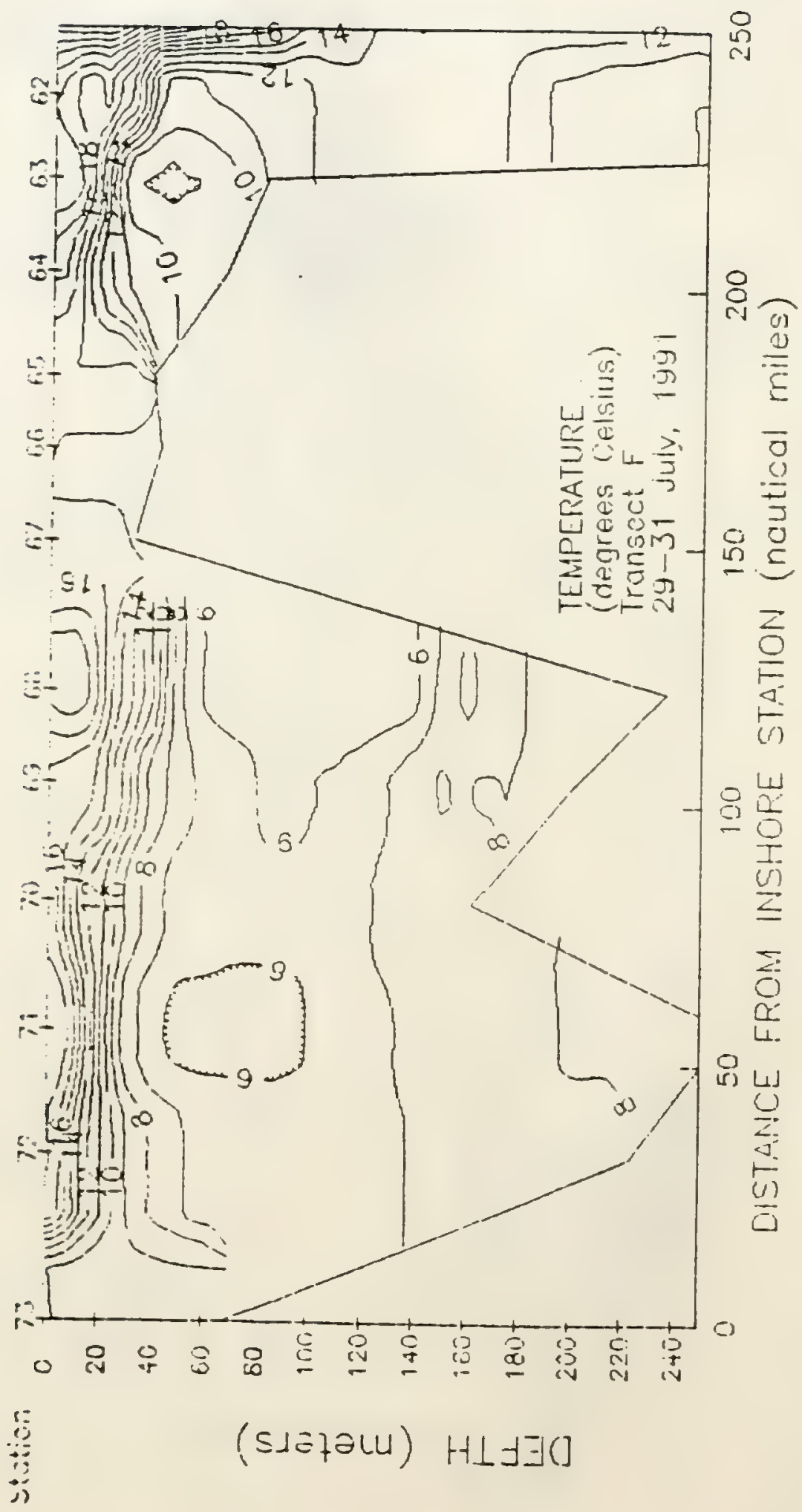




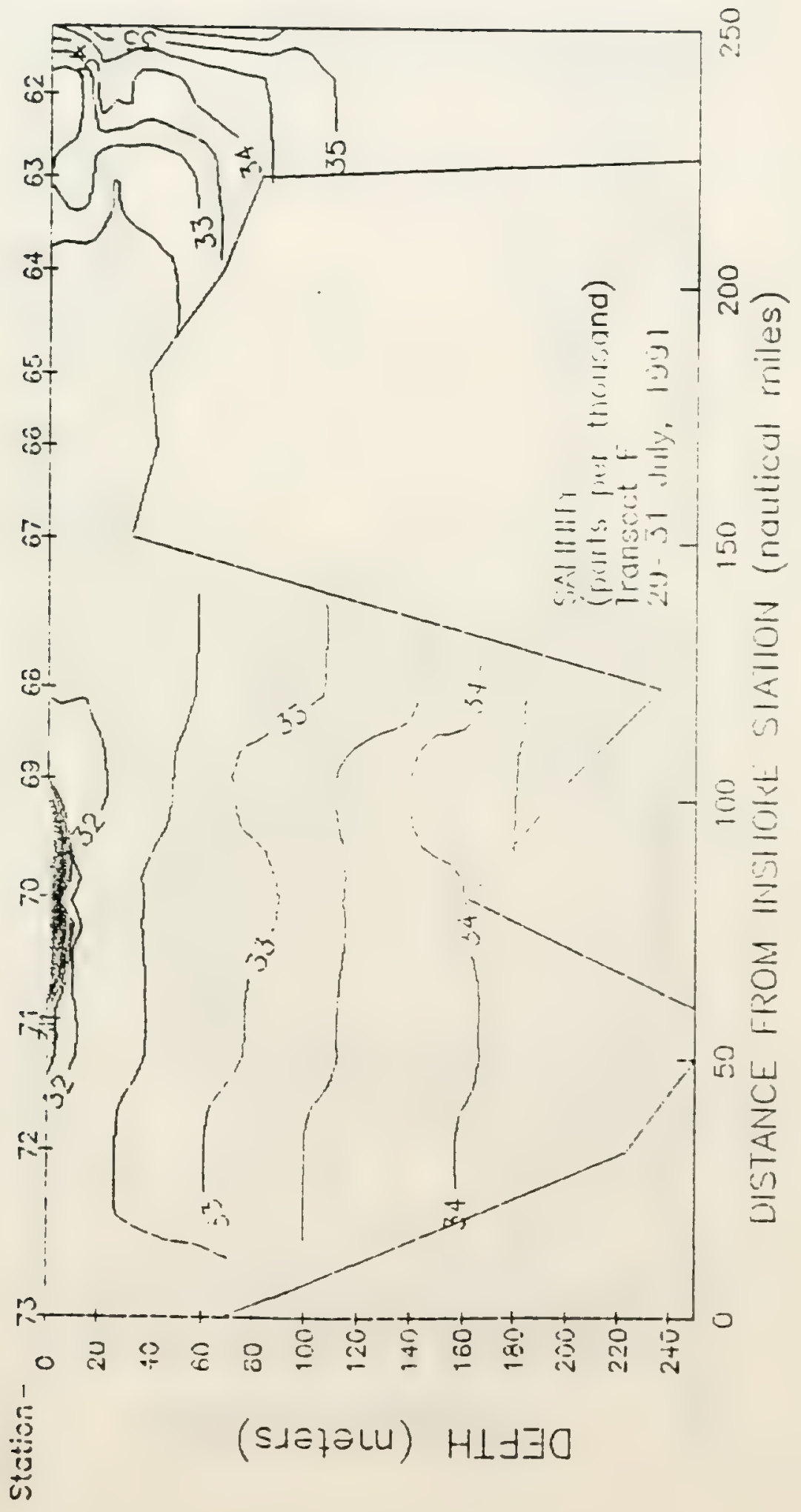




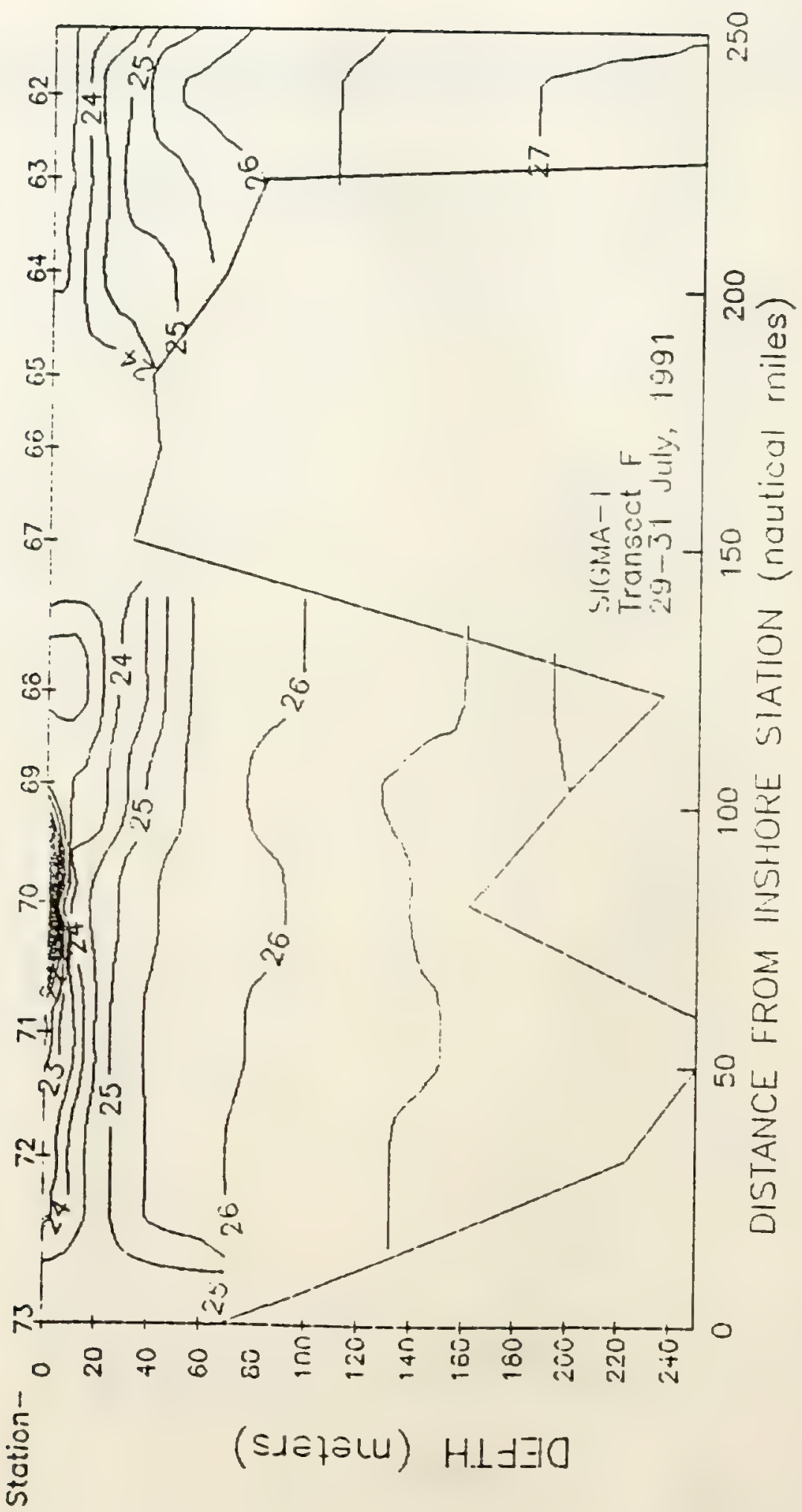




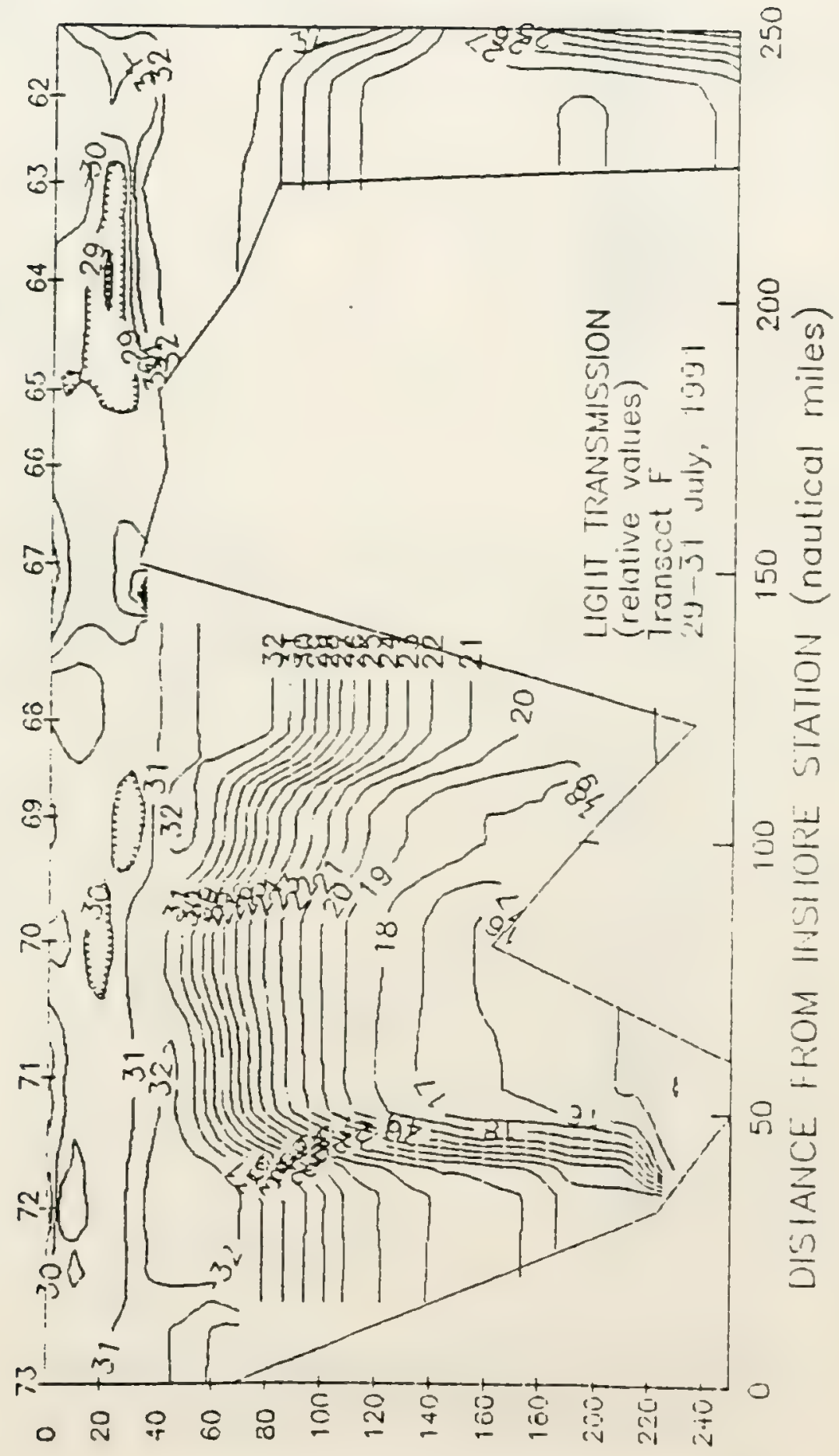

膏

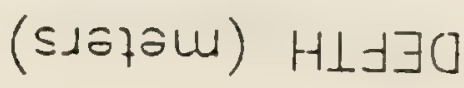




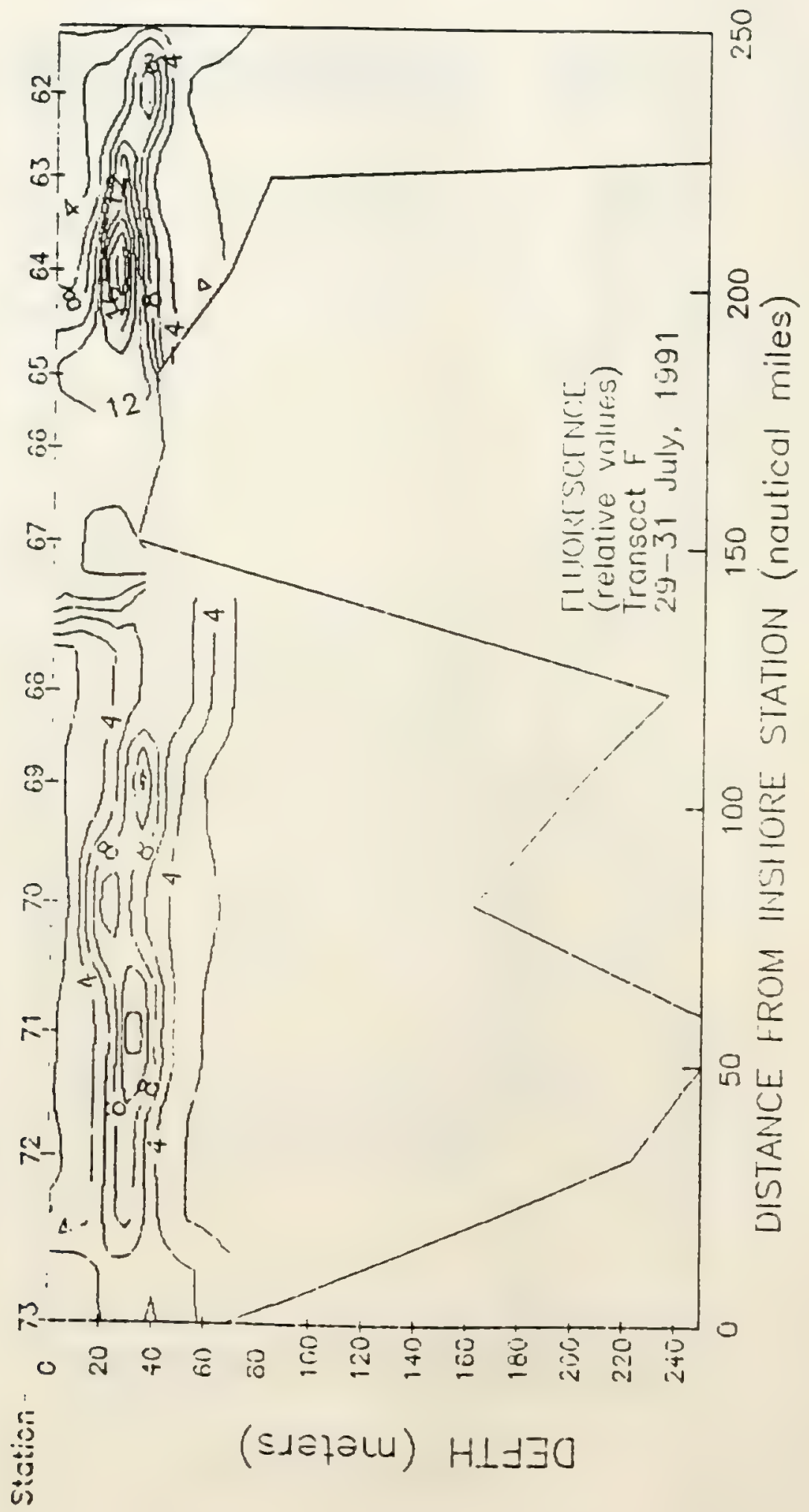




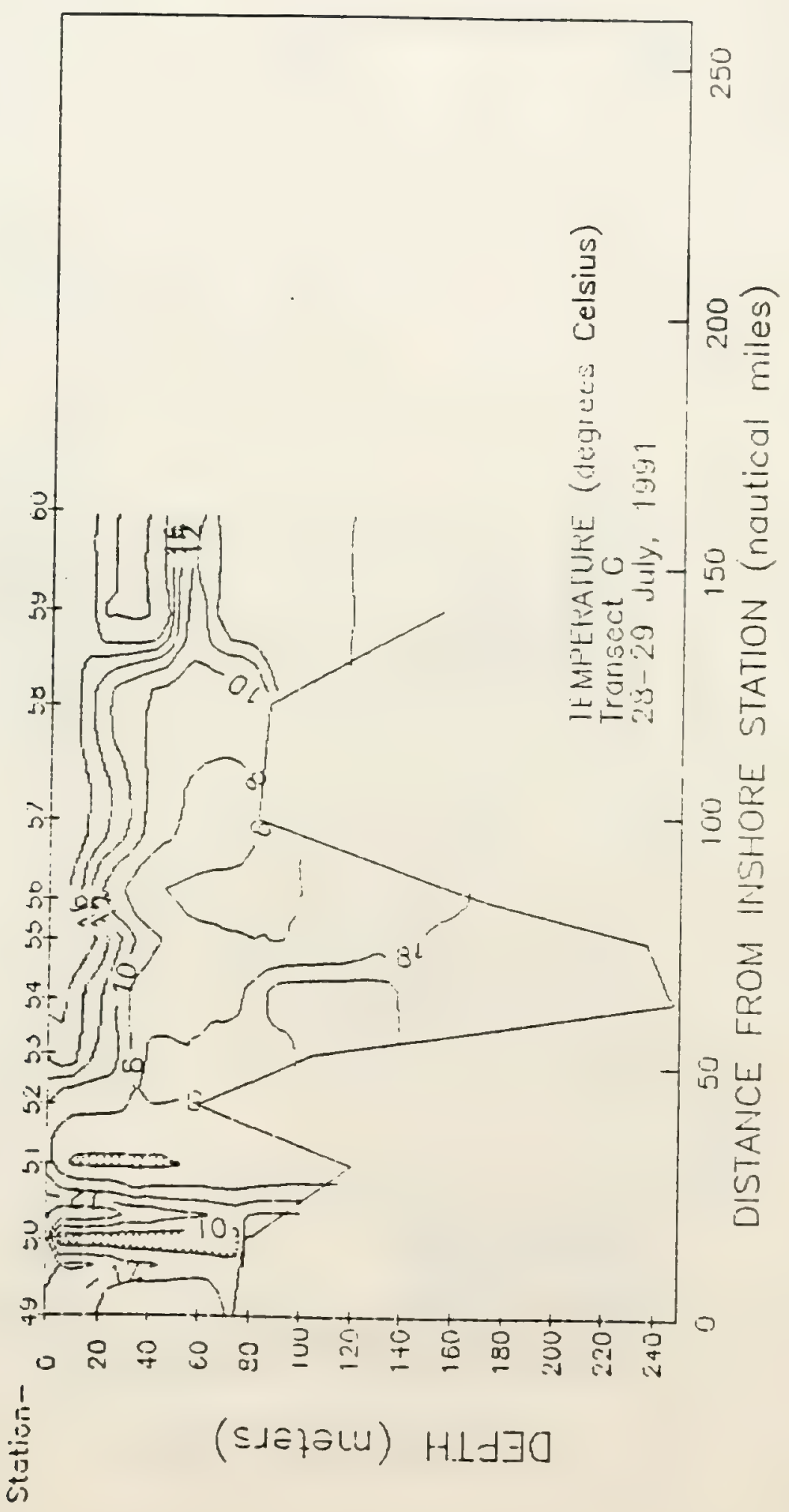




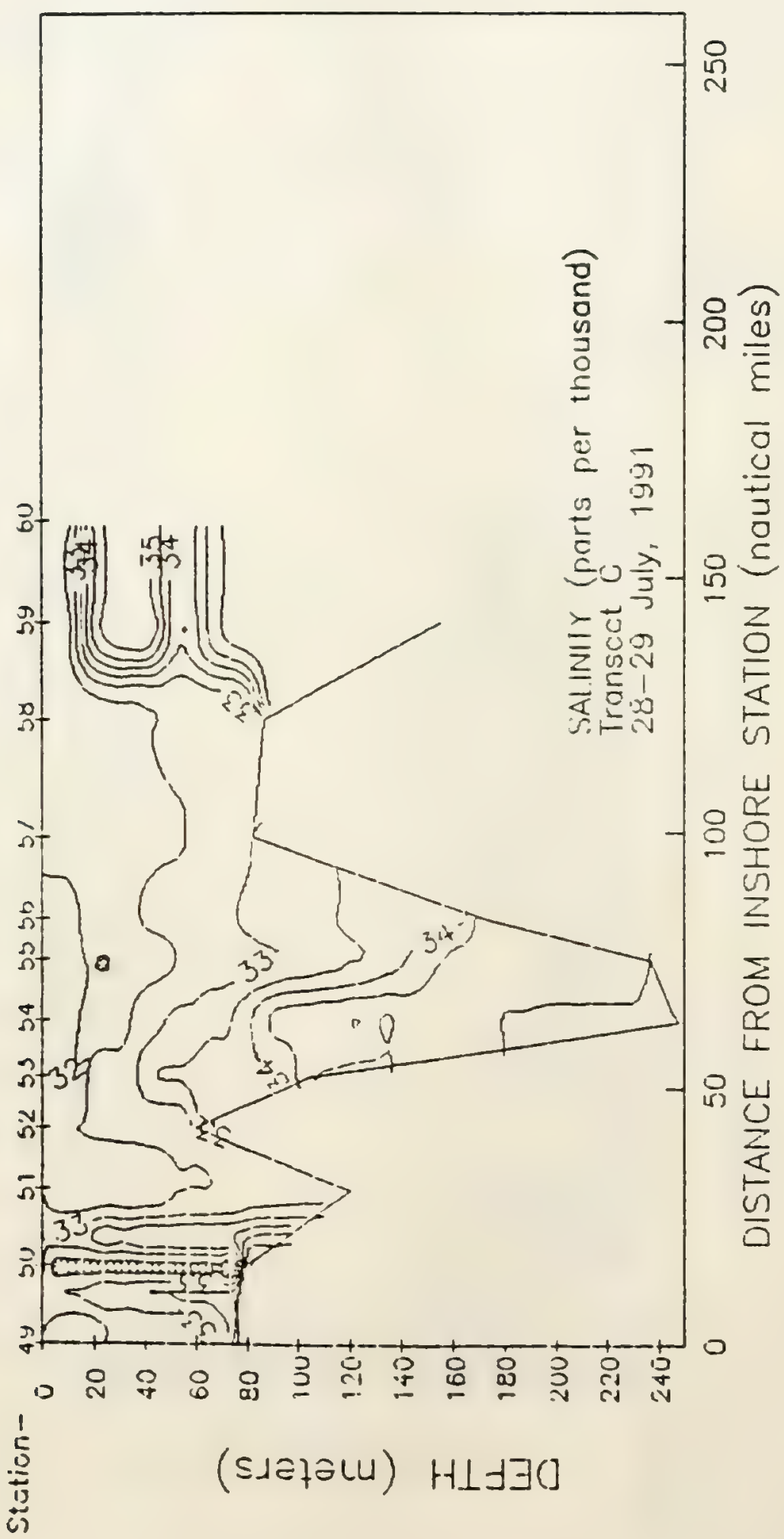




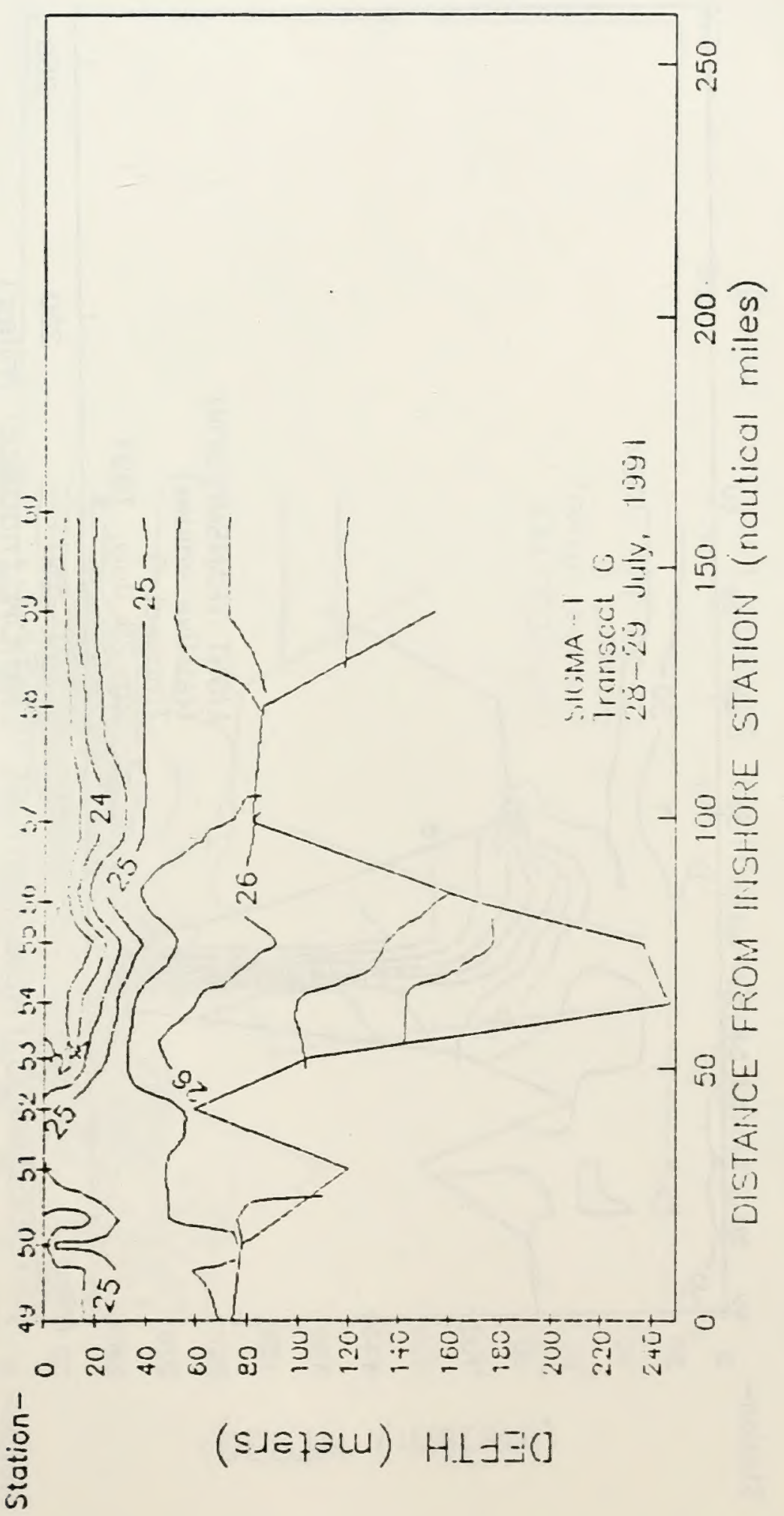




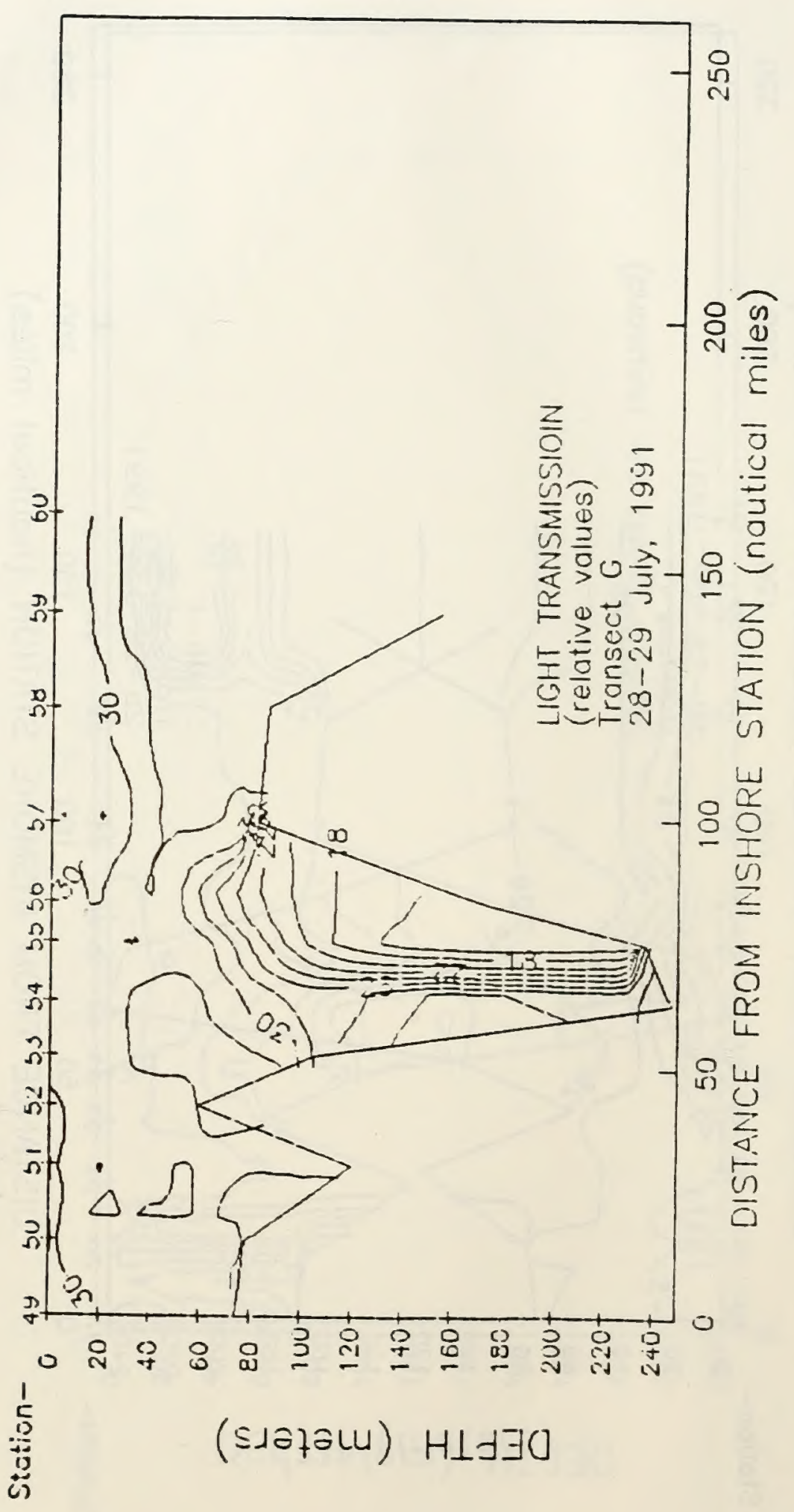




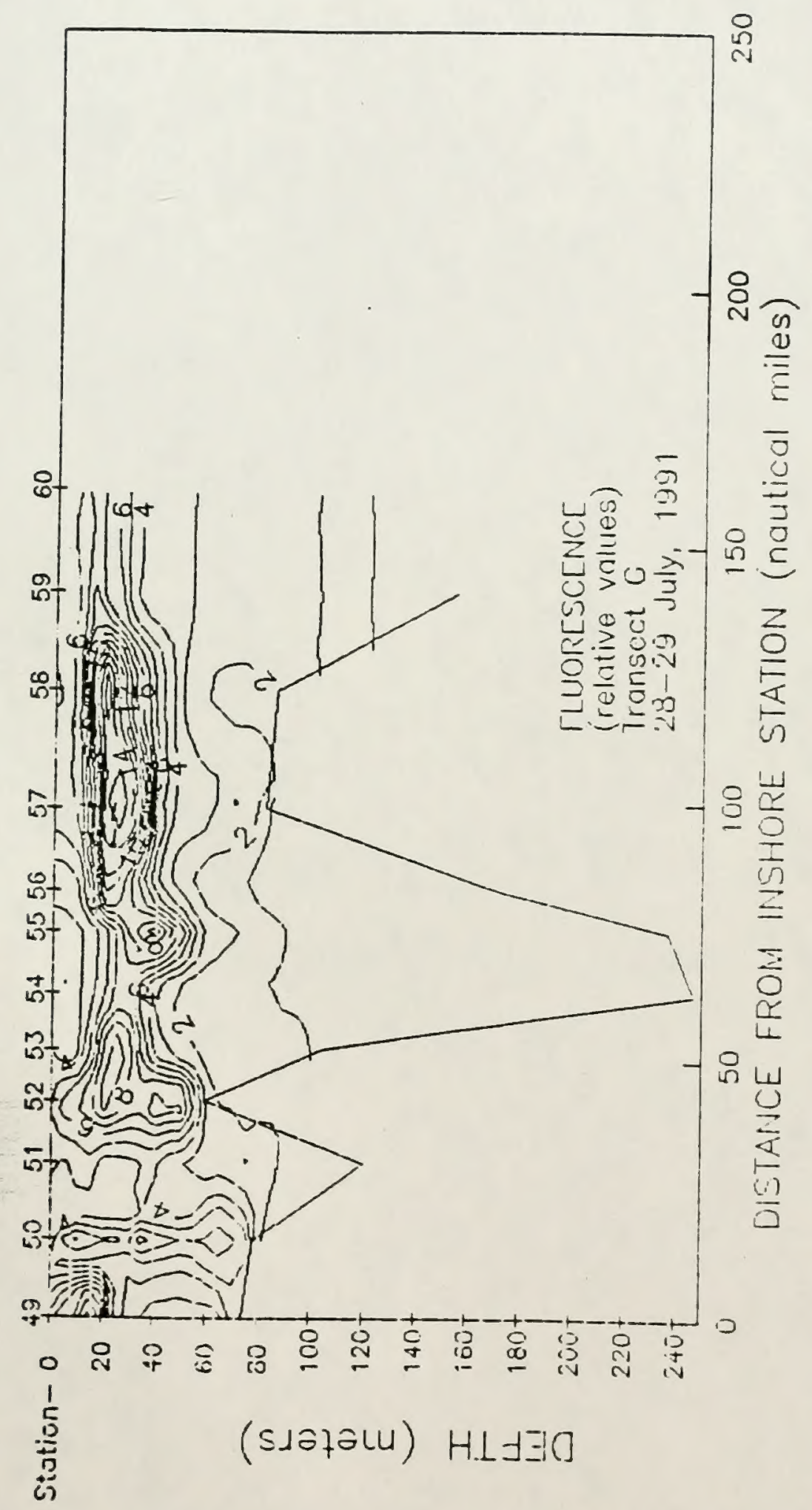


